\title{
Structural basis for specific inhibition of the highly sensitive ShHTL7 receptor
}

\author{
Umar Shahul Hameed ${ }^{1, \dagger}$ (D), Imran Haider ${ }^{2, \dagger}$, Muhammad Jamil ${ }^{2}$, Boubacar A Kountche ${ }^{2}$, Xianrong \\ Guo $^{3}$, Randa A Zarban², Dongjin Kim², Salim Al-Babili, ${ }^{2}$ (D) \& Stefan T Arold ${ }^{1, * *}$ (D)
}

\begin{abstract}
Striga hermonthica is a root parasitic plant that infests cereals, decimating yields, particularly in sub-Saharan Africa. For germination, Striga seeds require host-released strigolactones that are perceived by the family of HYPOSENSITIVE to LIGHT (ShHTL) receptors. Inhibiting seed germination would thus be a promising approach for combating Striga. However, there are currently no strigolactone antagonists that specifically block ShHTLs and do not bind to DWARF14, the homologous strigolactone receptor of the host. Here, we show that the octyl phenol ethoxylate Triton X-100 inhibits $S$. hermonthica seed germination without affecting host plants. High-resolution X-ray structures reveal that Triton X-100 specifically plugs the catalytic pocket of ShHTL7. ShHTL7-specific inhibition by Triton X-100 demonstrates the dominant role of this particular ShHTL receptor for Striga germination. Our structural analysis provides a rationale for the broad specificity and high sensitivity of ShHTL7, and reveals that strigolactones trigger structural changes in ShHTL7 that are required for downstream signaling. Our findings identify Triton and the related 2-[4-(2,4,4-trimethylpentan-2-yl) phenoxy]acetic acid as promising lead compounds for the rational design of efficient Striga-specific herbicides.
\end{abstract}

Keywords hydrolase; MAX2; seed germination inhibitor; Striga hermonthica; X-ray crystallography

Subject Categories Microbiology, Virology \& Host Pathogen Interaction; Plant Biology; Structural Biology

DOI 10.15252/embr.201745619| Received 9 December 2017| Revised 19 June 2018 | Accepted 20 June 2018 | Published online 18 July 2018

EMBO Reports (2018) 19: e45619

\section{Introduction}

The witchweed Striga hermonthica (Orobanchaceae) is a root parasitic plant that infests cereals, including rice, maize, sorghum, and pearl millet [1]. This parasitism stunts the host's growth, decimating yields, particularly in sub-Saharan Africa. By causing around 7 billion dollars annual loses in cereals production, Striga has become one of the most serious biotic threats to food security [2]. Related species with different host specificity increasingly impact agriculture in the temperate areas of Europe, the Middle East and North Africa, and Asia [3].

The germination of Striga seeds depends on host-released strigolactones (SLs) [1]. SLs are carotenoid-derived phytohormones that shape plants in accordance with nutrient availability [4-7]. SLs consist of a butenolide ring (D-ring) linked to a second, more variable moiety via an enol ether bridge [7-9]. The SL receptor, DWARF14 (D14) in rice, is a $\alpha / \beta$ hydrolase [9-12]. Hydrolysis of SLs by D14 is mediated by a conserved Ser-His-Asp catalytic triad and results in covalent linking of the D-ring to the receptor and the release of the second moiety [9-13]. This covalent modification promotes the interaction of D14 with the downstream effector MORE AXILLARY GROWTH 2 (MAX2), which requires substantial restructuring of the SL binding pocket of D14 to fit the MAX2 binding surface [12-14] and leads to the proteasome-mediated degradation of presumed repressors of SL-inducible genes, such as the rice DWARF53 [15-17]. The protein KARRIKIN-INSENSITIVE2 (KAI2)/ HYPOSENSITIVE to LIGHT (HTL)/D14-like is a close homolog to D14, which, however, binds the smoke-derived karrikins $[17,18]$ that induce seed germination in many land plants, but not in root parasitic plants [17].

Striga is an obligate parasite that cannot survive without a host, to which it needs to connect via a haustorium developed shortly after germination [19]. Hence, the perception of host SLs is a crucial step in Striga's life cycle, which ensures the presence of a host and determines whether imbibed seeds can develop to adult parasites. Striga hermonthica has a set of SL receptors, among which ShHTL7 is by far the most sensitive. These receptors evolved from extensive KAI2 gene duplication in Orobanchaceae and new functionalization toward sensing SLs instead of karrikins [20-22].

Striga produces up to 200,000 tiny seeds per plant, which can be easily dispersed and remain viable for more than a decade [23]. The accumulation of these seeds has led to heavy soil infestation,

\footnotetext{
1 Division of Biological and Environmental Sciences and Engineering, Computational Bioscience Research Center, King Abdullah University of Science and Technology, Thuwal, Saudi Arabia

2 Division of Biological and Environmental Sciences and Engineering, The Bioactives Lab, King Abdullah University of Science and Technology, Thuwal, Saudi Arabia

3 Core Labs, King Abdullah University of Science and Technology, Thuwal, Saudi Arabia

*Corresponding author. Tel: +966 1280 82565; E-mail: salim.babili@kaust.edu.sa

${ }^{* *}$ Corresponding author. Tel: +966 1280 82557; E-mail: stefan.arold@kaust.edu.sa

'These authors contributed equally to this work
} 
making Striga control onerous. As a wild, non-domesticated species, the maturation of Striga seeds is not synchronized, resulting in a seed population where only a subset is ready to germinate while others are in an inert dormant state. Moreover, most of the damage by Striga occurs invisibly below ground before the weed emerges from the soil. Hence, successful Striga control strategies must include coping with this heterogeneous seed reservoir accumulated in the soil. Suicidal germination is a possible approach, which is based on inducing seed germination by applying SL analogs in the absence of a host, thus killing Striga seedlings without affecting the host's SL homeostasis [24,25]. However, this method can be only used in a narrow, difficult to determine time window that allows catching the germination-ready Striga seeds before sowing crop seeds. Striga seeds that mature after treatment remain unaffected and can still decimate the crops. A very promising complementary approach is to inhibit the germination of Striga seeds by applying antagonists that block Striga SL perception $[26,27]$. If such inhibitors do not bind to host SL receptors, they can be applied in the presence or absence of the host as specific herbicides that enable Striga control throughout the growing season. However, such specific inhibitors are currently not available. Moreover, an experimental 3D protein structure of ShHTL7 that allows specific in silico screening for inhibitors is still missing.

\section{Results}

\section{Structural basis of Triton binding to ShHTL7}

We obtained 1.2-1.7- $\AA$ resolution X-ray diffraction data from crystals

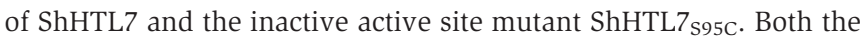
wild-type and mutant proteins were crystalized either without a ligand or soaked with the SL analog GR24 (Table 1). We determined their structures by molecular replacement, using the ShHTL5 structure as a template [21]. Surprisingly, in all crystals, ShHTL7 associated with the 4-(1,1,3,3-tetramethylbutyl)-phenyl hydrocarbon moiety of Triton X-100 that we used during protein purification (Figs $1 \mathrm{~A}$ and $\mathrm{B}$ and EV1A). Isomorphous crystal structures of ShHTL7 purified without using Triton exhibited empty binding pockets (Fig EV1B), confirming the source and identity of this compound.

Triton bound to the deep active site pocket, formed by a double V-shaped arrangement of helices $\alpha 4-\alpha 5$ and $\alpha 6-\alpha 7$. The hydrocarbon moiety of Triton established hydrophobic interactions with Y26 (loop following $\beta 2$ ), L124, F134 ( $\beta 5-\alpha 4$ loop), M139, T142, L143, L146 (on $\alpha 4$ ), F150 ( $\alpha 4-\alpha 5$ loop), L153, T157, L161 ( $\alpha 5)$, T190, I193, C194 ( $\alpha 7$ ), and M219 ( $\beta 6-\alpha 8$ loop; Figs 1C-E and EV2). These residues also constitute the SL binding site, as inferred by homology to rice D14 [11]. However, Triton remained at a distance of more than $8 \AA$ from the catalytic serine $\mathrm{S} 95$ in the active site (Fig 1C). In ShHTL7 $7_{\mathrm{S} 95 \mathrm{C}}$, the sulfhydryl group in $\mathrm{C} 95$ was oxidized to a sulfinic acid, as confirmed by mass spectrometry (Fig EV3), causing only a minor rearrangement of the $\mathrm{H} 94$ side chain in otherwise identical structures (RMSD of $0.108 \AA$; Appendix Fig S1). Well-ordered water molecules filled the space between Triton and the bottom of the active site pocket (Fig 1C). These water molecules were well preserved in crystals of ShHTL7 purified without Triton, showing that Triton binding did not displace or rearrange this pre-existing water network.
The Triton polyethylene head group protruded out of the active site pocket (Fig 1A-D). Five ethylene oxide units were visible in a relatively weak and poorly defined electron density, suggesting weak association of this moiety. The headgroup-ShHTL7 interactions with the outer rim of the ShHTL7 binding pocket comprised a watermediated hydrogen bond (from the oxide of the first unit to the backbone nitrogen of E135) and hydrophobic contacts (between carbons of the first unit and a hydrophobic pocket formed by ShHTL7 V138, M139, and T142, and between carbons of the fourth unit and L160 and I218). This orientation might be influenced by crystal contacts, which occlude part of the accessible protein surface.

\section{ShHTL7 binds strongly and specifically to Triton in vitro}

A time course of intrinsic Triton fluorescence assays revealed a very slow association for both, ShHTL7 and the inactive

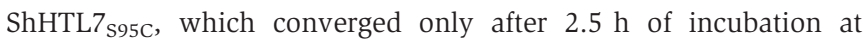
dissociation constants $\left(K_{\mathrm{d}}\right)$ of $284 \pm 22 \mathrm{nM}$ and $195 \pm 21 \mathrm{nM}$, respectively (Fig $2 \mathrm{~A}$ and $\mathrm{B}$ ). These experiments were performed in a standard buffer containing $0.005 \%$ of the detergent Tween-20. In the absence of Tween-20, ShHTL7 bound to Triton with a final $K_{\mathrm{d}}$ of $233 \pm 30 \mathrm{nM}$, showing that the presence of Tween-20 did not significantly lower the affinity (Fig EV4A). The fact that the Triton-ShHTL7 complex withstood size exclusion chromatography and extensive dialysis corroborated the high affinity of the Triton association, despite the apparent slow on-rate. ShHTL7 also exhibited aggregation temperature $\left(T_{\mathrm{agg}}\right)$ increases in a Triton concentration-dependent manner after $2.5 \mathrm{~h}$ of pre-incubation (Fig 2C). Conversely, n-dodecyl- $\beta$-D-maltoside (nDM, a structurally different detergent with a critical micellar concentration similar to that of Triton) did not increase the protein $T_{\text {agg }}$ under the same conditions (Fig EV4B). To further quantitate the binding, we used microscale thermophoresis measurements (MST) after $2.5 \mathrm{~h}$ of Triton preincubation, which resulted in a dissociation constant $\left(K_{\mathrm{d}}\right)$ of $0.44 \pm 0.05 \mu \mathrm{M}$ (Fig 2D). We carried out a drug affinity response target stability (DARTS) assay to establish whether Triton could stabilize ShHTL7 in the presence of proteases, similarly to GR24 [26]. Both Triton and GR24 efficiently protected the protein from being digested by proteinase $\mathrm{K}$, whereas point mutants that specifically lack binding to either GR24 or Triton failed to protect ShHTL7 from digestion (Fig EV4C).

\section{Triton efficiently blocks binding of SLs to ShHTL7}

ShHTL7 bound Triton with a slightly stronger affinity than GR24 (apparent $K_{\mathrm{d}}$ of GR24 was $0.92 \pm 0.01 \mu \mathrm{M}$ in MST, and $0.39 \pm 0.05 \mu \mathrm{M}$ in tryptophan fluorescence assays; Fig $2 \mathrm{E}$ and $\mathrm{F}$ ). To test whether Triton inhibits SL binding, we performed a competition assay using Yoshimulactone Green (YLG) as SL ligand. In the absence of Triton, $3 \mu \mathrm{M}$ of ShHTL7 hydrolyzes $1 \mu \mathrm{M}$ of YLG within 50 min (Appendix Fig S2A). Pre-incubation with Triton for $2.5 \mathrm{~h}$ blocked ShHTL7 hydrolysis activity with an $\mathrm{IC}_{50}$ of $0.47 \pm 0.11 \mu \mathrm{M}$, which is about half the $\mathrm{IC}_{50}$ of GR24 $(0.87 \pm 0.06 \mu \mathrm{M}$; Fig $2 \mathrm{G}$ and $\mathrm{H}$ ), and 25 -fold lower than that reported for the non-specific inhibitor Soporidine [26]. The $\mathrm{IC}_{50}$ value for GR24 obtained in our YLG assay is comparable to previously published YLG assays values [21]. Interestingly, the $\mathrm{EC}_{50}$ of GR24 for ShHTL7 in transgenic Arabidopsis is in the picomolar range [22], suggesting that unknown 
Table 1. X-ray diffraction data collection and refinement statistics for ShHTL7.

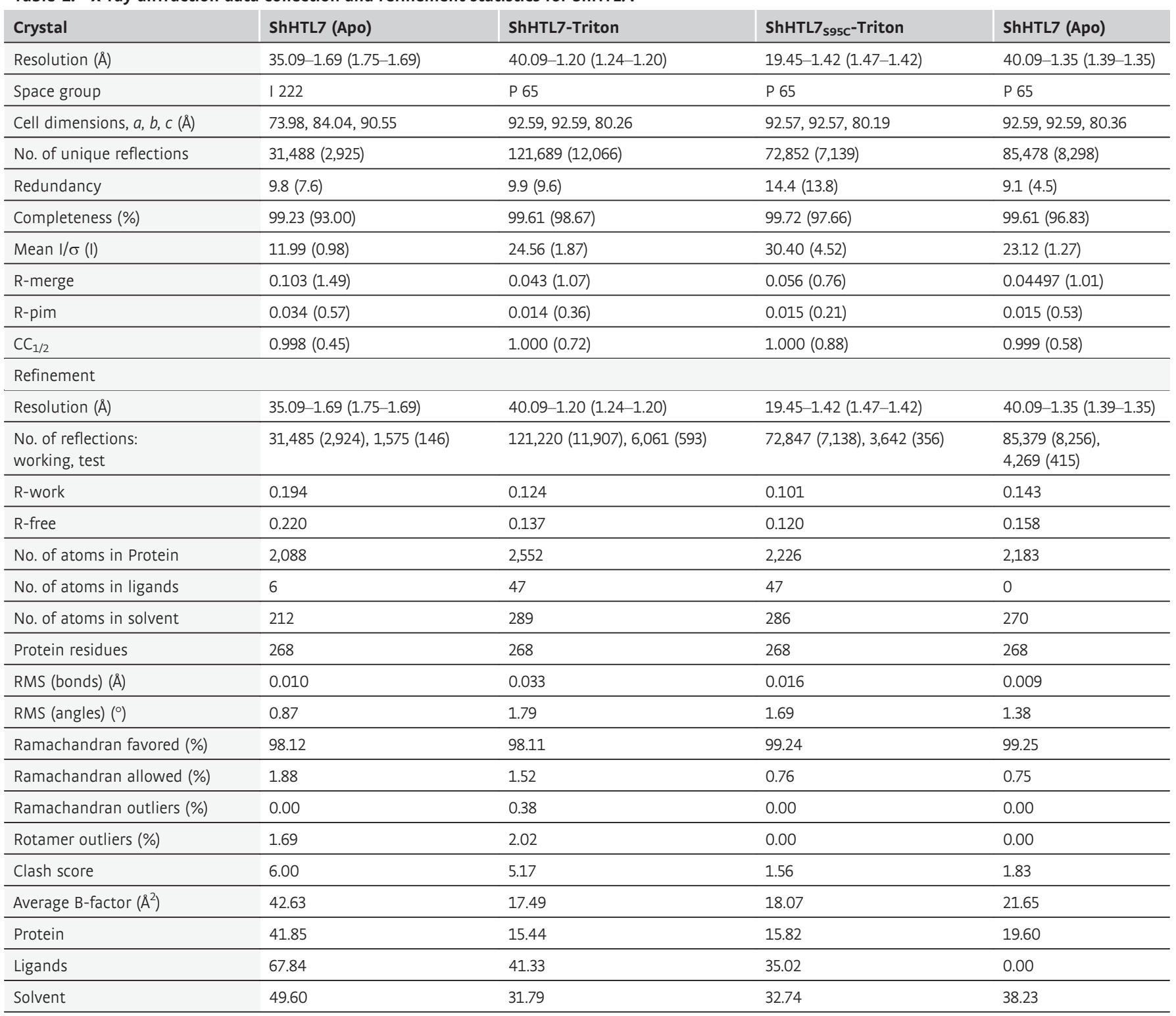

Values in parentheses are for the highest resolution shell.

additional factors may play a role in this in vivo assay (e.g., local enrichment or co-factors).

\section{Triton blocks structural changes and downstream signaling of ShHTL7 induced by GR24}

Triton-free ShHTL7 structures obtained in the same space group (P65) as the Triton-bound structures did not exhibit significant changes in the binding site (Appendix Fig S2B). However, the P65 crystal packing implicated helices $\alpha 4$ and $\alpha 5$ of the binding pocket and may have stabilized the Triton-binding conformation even in the apostate. Indeed, apo-ShHTL7 obtained from a different crystal form (I222) where $\alpha 4$ is not constrained, showed that $\alpha 4$ had moved inward by about $2 \AA$, as compared to the P65 structures (Fig 2I). In this conformation, a steric overlap with $\alpha 4$ residue T142 precludes
Triton binding, strongly suggesting that the rate-limiting factor for Triton binding is the opening of $\alpha 4$. In solution nuclear magnetic resonance (NMR) spectroscopy, the changes in position and/or intensity of several well-dispersed chemical shifts between apo ${ }^{15} \mathrm{~N}$ labeled ShHTL7 and ${ }^{15} \mathrm{~N}$-ShHTL7 purified in the presence of Triton were of the amount and magnitude expected from our crystallographic analysis (Figs 3A and EV5). This also unequivocally confirms the direct residue-specific interaction between Triton and ShHTL7 in solution, without any major conformational changes from the protein, in line with our crystallographic analysis. Smallangle X-ray scattering (SAXS) data of apo-ShHTL7 and ShHTL7 incubated with Triton were highly similar (Appendix Fig S3A and B; radius of gyration, $R_{g}$, were $19.7 \pm 0.2 \AA$ and $19.5 \pm 0.2 \AA$ for apo and Triton-bound forms, respectively) and fitted the experimental structures very well (Appendix Fig S3A and B; $\chi^{2}$ were 2.46 and 
A

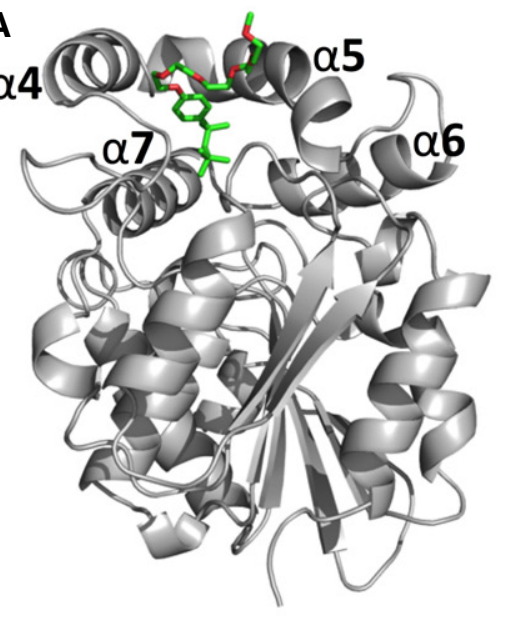

B

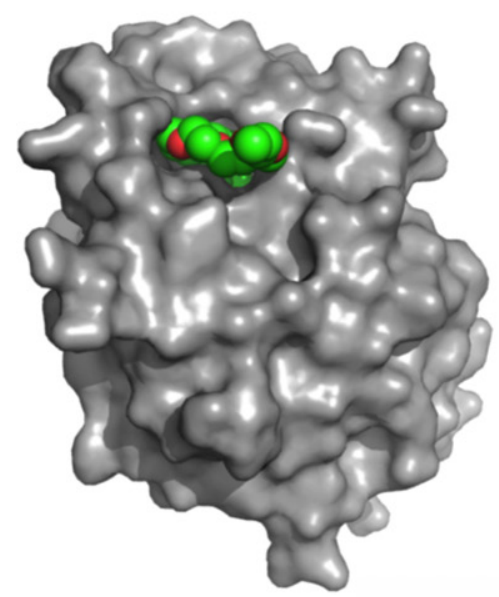

C

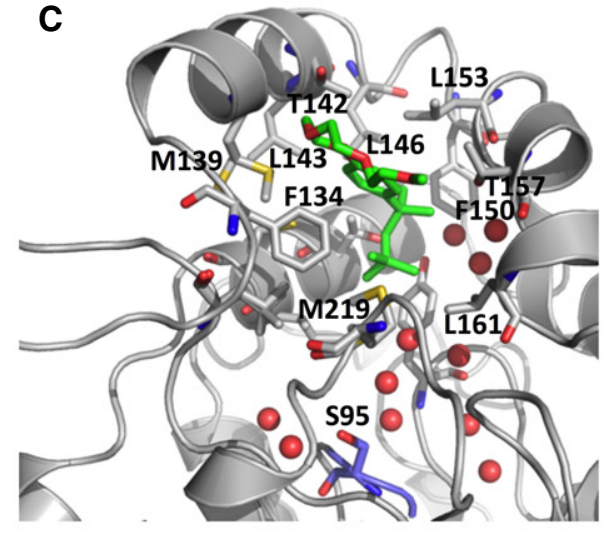

D

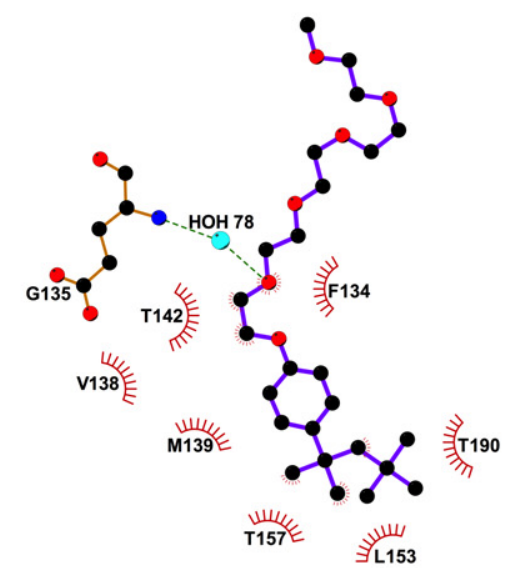

E

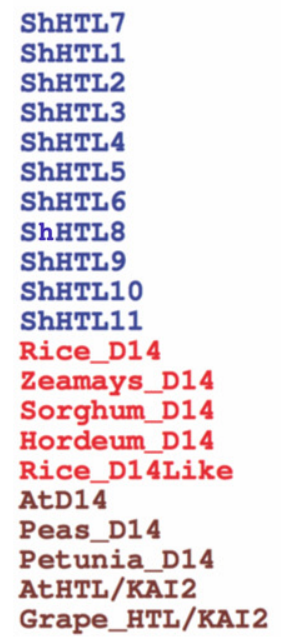

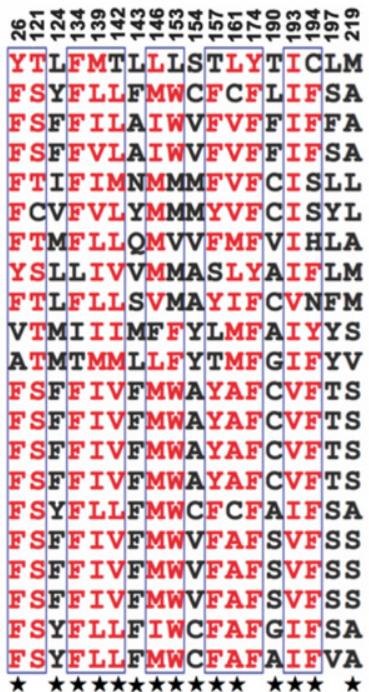

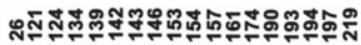
YTIFMTLLISTLYTICLM F SYF LIFMW CF CFIIFSA F SFF IIAIWVFVFFIFFA F SFFVIAI WVFVFFIFSA F TIF IMNMMMFVF CI SIL FCVFVL YMMMYVFCI SYL FTMF LL QMVVFMFVI HLA YSLI IVVMMASL YA I F LM F TLF LI SVMAY I F CVNFM VTMI I IMF FY LMFA I YYS ATMTMMILFYTMF GIFYV F SFF IVFMWAYAF CVFTS FSFF IVFMWAYAFCVFTS F SFF IVFMWAYAF CVFTS F SFF IVFMWAYAFCVFTS F SYF LIFMWCFCFAIFSA F SFF IVFMWVFAF SVFSS F SFF IVFMWVFAFSVFSS IVIW AFSUSS FSYFLIFMWCFAFAIFVA

Figure 1. Mechanism of Triton binding to ShHTL7.

A Crystal structure of ShHTL7 (gray) bound to Triton (stick model; green: carbons; red: oxygens).

B Surface (protein) and sphere (Triton) representation of (A) tilted by $\sim 10^{\circ}$ toward the viewer.

C Zoom into the ShHTL7 binding pocket. Key side chains and Triton are shown as stick models. Red spheres depict ordered water molecules.

D LigPlot of the ShHTL7-Triton interactions. Hydrophobic interactions: red arc with spokes radiating toward the ligand atoms involved; hydrogen bonds: dashed lines.

E Multiple sequence alignment of the ShHTL7 SL binding site against homologs from various species. Red residues: similar stereochemical character. Boxed: highly conserved positions. Residues marked with a star contribute to Triton interactions. For a full alignment, see Fig EV2.

$3.8 \AA$ for apo and Triton-bound ShHTL7, respectively). Collectively, these analyses validated our crystallographic analysis in solution and ruled out major structural changes upon Triton-binding.

Conversely, the titration of ${ }^{15} \mathrm{~N}$-ShHTL7 with GR24 resulted in much more pronounced chemical shift changes (Fig 3B). Pre-incubation of ${ }^{15} \mathrm{~N}$-ShHTL7 with Triton before adding GR24 precluded these chemical shift changes, supporting that Triton can effectively block GR24induced structural changes in ShHTL7 (Fig 3C and Appendix Fig S3C). Accordingly, pre-incubation of ShHTL7 with Triton efficiently blocked the $T_{\text {agg }}$ changes in ShHTL7 that were otherwise introduced by GR24 in a concentration-dependent manner (Fig 3D). Of note, GR24 binding destabilized ShHTL7 ( $T_{\mathrm{agg}}$ changes in the presence of GR24 are negative), whereas Triton binding stabilized ShHTL7 (Fig 3D).

Structural analysis of the Arabidopsis thaliana GR24-D14 in a complex with the downstream activator AtMAX2 showed that a rearrangement of D14 helices $\alpha 5$ and $\alpha 6$, resulting in the collapse of the ligand-binding pocket, is necessary for an association with MAX2 [11]. Homology modeling of the ShHTL7:ShMAX2 complex based on the A. thaliana D14:MAX2 structure showed that the interface is conserved (13 residues are identical, one is homologous in ShMAX2, and all 14 residues are identical in ShHTL7; ShMAX2 and AtMAX2 share $79 \%$ sequence identity in the binding site; Appendix Fig S4), suggesting that ShHTL7 requires the same type of structural rearrangements as D14 to bind to its cognate MAX2 molecule (Appendix Fig S5A-C). SAXS data recorded on ShHTL7 incubated with GR24 showed indeed a decrease in the characteristic SAXS peak between $q=0.2$ and $0.35 \AA-1$, as expected from a collapse of the large active site pocket in the ShMAX2-bound form of ShHTL7 (Appendix Fig S3C). Our observation that GR24-binding decreased the $T_{\text {agg }}$ of ShHTL7 further supported that GR24 destabilizes the ShHTL7 structure. Also, our NMR analysis of GR24-bound ShHTL7 was compatible with a loss of helix content in the SL-bound form and showed that this loss of 
A :

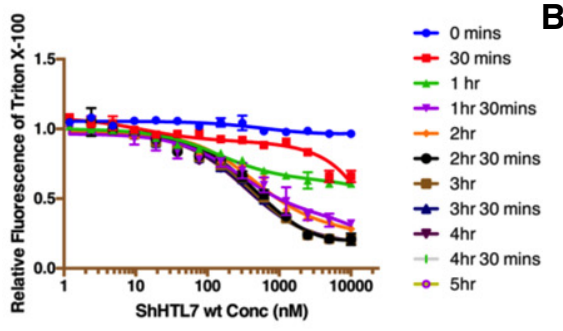

B

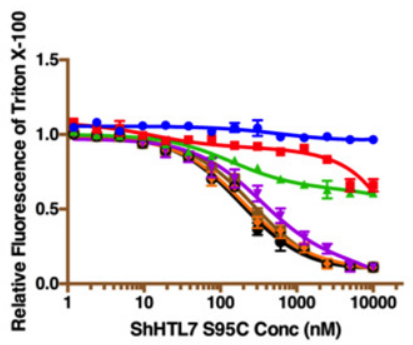

$\rightarrow 0$ mins

$-30 \mathrm{mins}$

$-1 \mathrm{hr}$

$\rightarrow 1 \mathrm{hr} 30 \mathrm{mins}$

$\rightarrow-1 \mathrm{hr} 30 \mathrm{hr}$

$\rightarrow 2 \mathrm{hr} 30 \mathrm{mins}$

$=3 \mathrm{hr}$

$+3 \mathrm{hr} 30 \mathrm{mins}$

$\rightarrow 4 \mathrm{hr}$

$+4 \mathrm{hr} 30 \mathrm{mins}$

$-5 \mathrm{hr}$

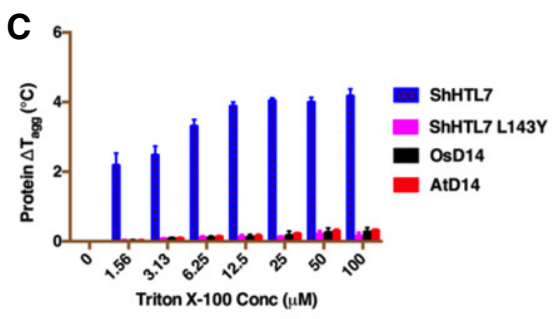

D
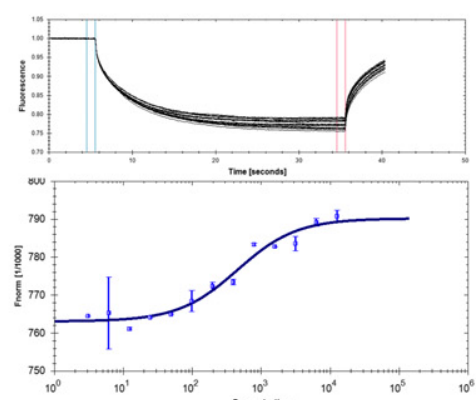

G

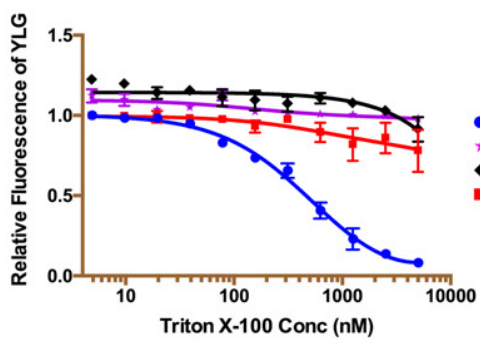

E
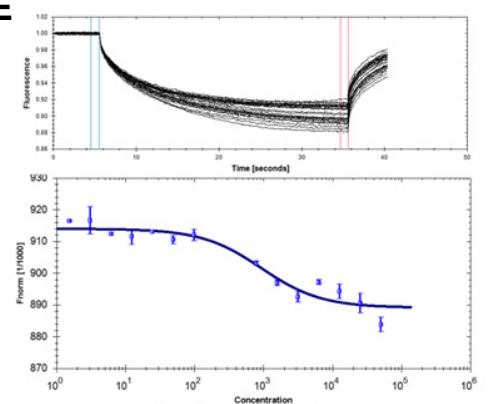

H

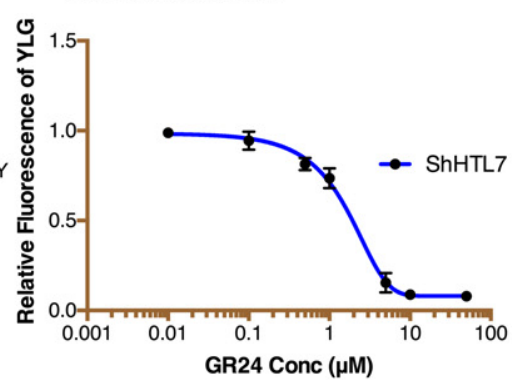

$\mathbf{F}$

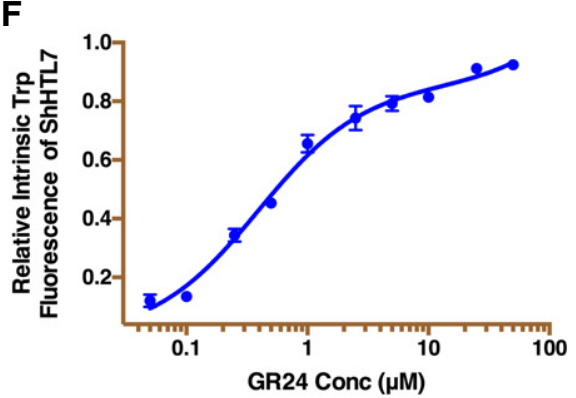

I $\alpha 4$

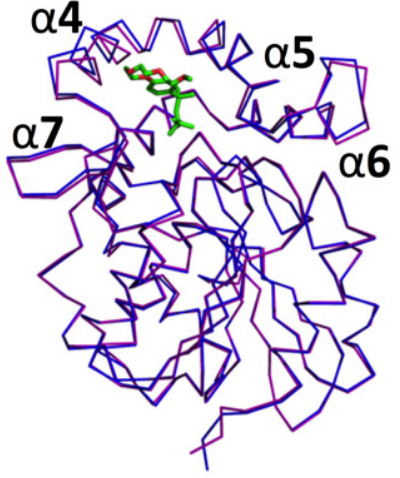

Figure 2. In vitro evaluation of Triton binding.

A, B Relative intrinsic Triton fluorescence for different incubation times, plotted against wild-type ShHTL7 concentrations (A) and ShHTL7 S95C mutant concentrations (B). Data are mean \pm SD, $n=3$.

$\mathrm{C}$ The change in protein aggregation temperature $\left(\Delta T_{\text {agg }}\right)$ plotted against Triton concentrations. Data are mean $\pm \mathrm{SD}, n=3$.

D, E Thermophoresis data of fluorescently labeled ShHTL7 binding to different concentrations of Triton (D) and GR24 (E) from $25 \mu \mathrm{M}$ to sub-nanomolar range. Data are mean \pm SD of the fluorescence counts from the labeled protein, $n=3$.

F Relative intrinsic tryptophan fluorescence of ShHTL7 is plotted against GR24 concentrations.

$\mathrm{G}, \mathrm{H}$ YLG hydrolysis by ShHTL7, ShHTL7 ${ }_{\mathrm{L} 143 \mathrm{Y}}$, and D14 proteins in the presence of increasing Triton (G) and GR24 (H) concentrations. Data are mean \pm SD, $n=3$.

I Apo-ShHTL7 from the I222 space group (blue), superimposed onto the Triton-bound ShHTL7 crystallized in P65 (purple). Inward movement of $\alpha 4$ in I222 precludes binding of Triton (green) with r.m.s.d. of $0.25 \AA$.

helix content was blocked by Triton (Appendix Fig S6). Collectively, our analysis suggested that Triton can block the association of ShHTL7 with MAX2 even in the presence of GR24 by precluding the structural rearrangement of ShHTL7 necessary for binding to ShMAX2 (Fig 4A and Appendix Fig S6). Accordingly, pre-incubation of ShHTL7 with Triton inhibited the GR24-dependent interaction of ShHTL7 with the S. hermonthica downstream activator ShMAX2 (Fig 4B and Appendix Fig S5C and S6).

Triton blocks germination of Striga through ShHTL7, but does not affect rice plants

Next, we exposed different Striga seed batches to increasing concentrations of Triton and GR24. We observed a $50 \%$ reduction in GR24-induced germination in the presence of $15 \mu \mathrm{M}$ Triton. This result is not explained by a general inhibitory effect of detergent on Striga seeds, because under the same conditions, the application of the detergent $\mathrm{n}$-dodecyl- $\beta$-D-maltoside did not affect germination (Fig 4C). Three-dimensional homology modeling of other Striga SL receptors (ShHTL1-11) indicated specificity of Triton for ShHTL7 (Appendix Text S1). The active site composition of other ShHTLs, especially ShHTL5 and 6, appeared to preclude Triton binding because of steric clashes (in ShHTL6: T142L, T157F, and C194H; in ShHTL5: T142L and T157Y; Appendix Fig S7). Thus, the observed effect is expected to be solely caused by the inhibition of ShHTL7.

For field application, Striga seed germination inhibitors should not bind to ShHTL homologs in non-parasitic plants. We tested 
A

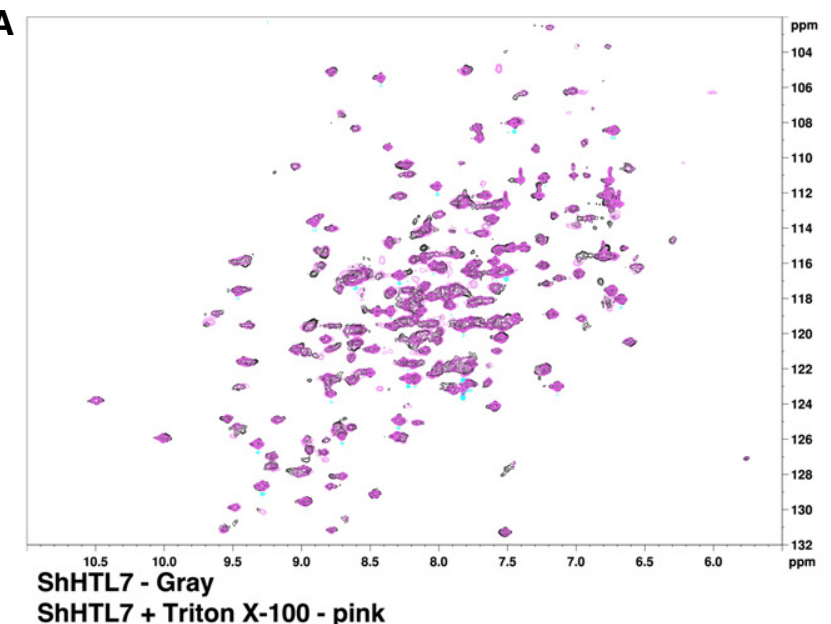

C

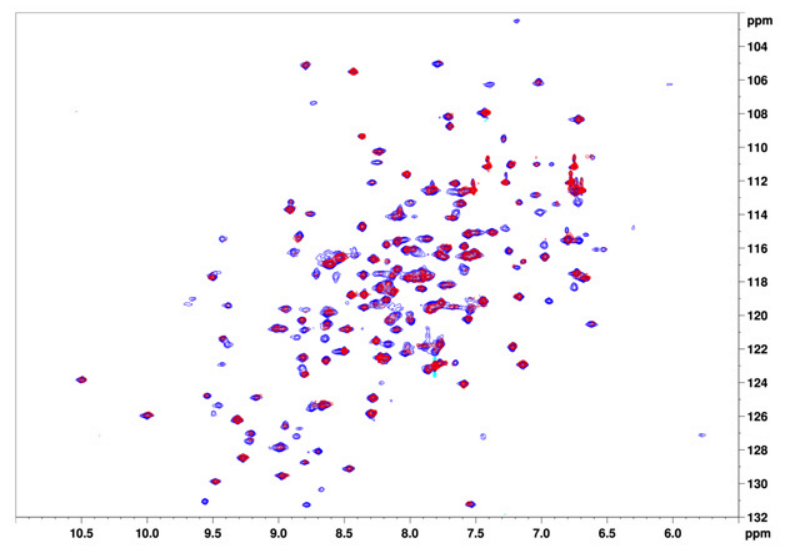

ShHTL7 + GR24 - Red

ShHTL7 + Triton X-100 + GR24 - Blue
B

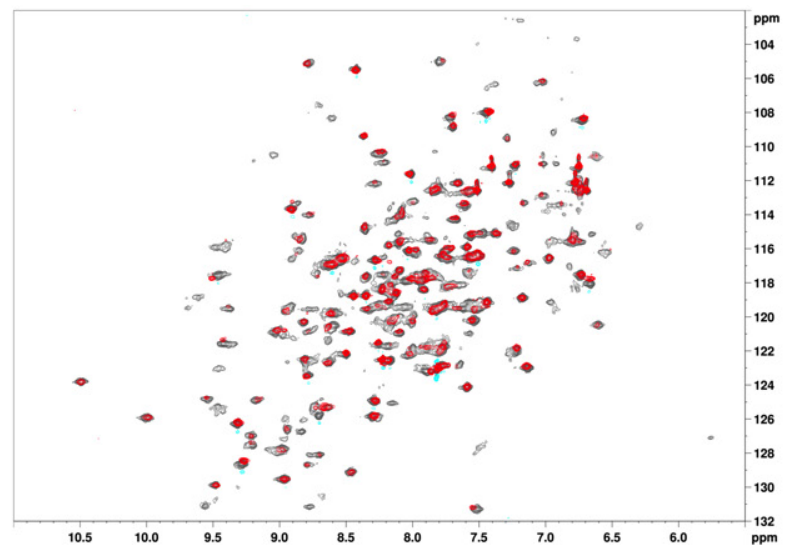

ShHTL7 - Gray

ShHTL7 + GR24 - Red

D

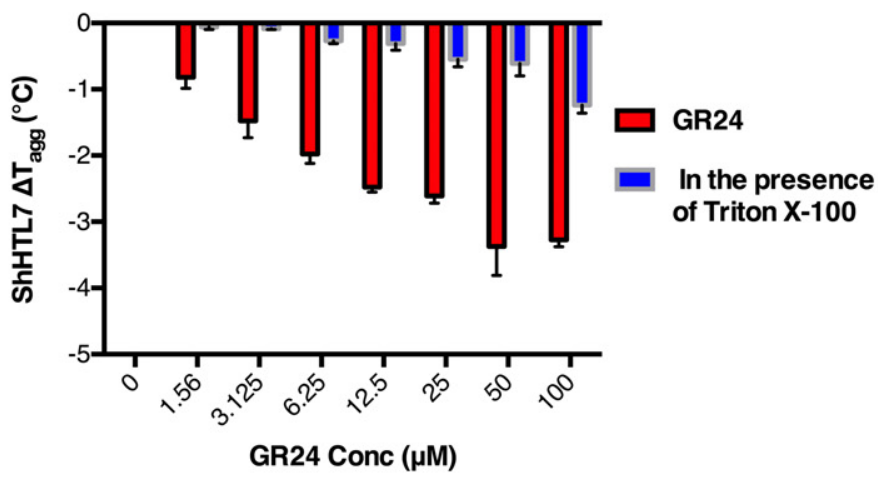

Figure 3. Triton binds to ShHTL7 and stabilizes the protein from structural changes.

A ${ }^{15} \mathrm{~N} \mathrm{HSQC}$ spectra of $0.3 \mathrm{mM} \mathrm{ShHTL7}$ (gray) overlapped on ShHTL7 purified in the presence of Triton (pink).

B ${ }^{15} \mathrm{~N} \mathrm{HSQC}$ spectra of $0.3 \mathrm{mM}$ ShHTL7 (gray) with spectra colored red at the final ShHTL7-GR24 ratio of 1:1.5.

C ${ }^{15} \mathrm{~N}$ HSQC spectra of $0.3 \mathrm{mM}$ ShHTL7 + Triton (dark blue) overlapped on ShHTL7 (red) at the final ShHTL7-GR24 ratio of 1:1.5.

$\mathrm{D}$ The change in ShHTL7 (red) and ShHTL7 + Triton (blue) aggregation temperature $\left(\Delta T_{\text {agg }}\right)$ plotted against GR24 concentrations. Data are mean \pm SD, $n=3$.

in vitro binding of Triton to Arabidopsis and rice D14 (AtD14 and OsD14, respectively). Triton fluorescence did not change in the presence of up to $5 \mu \mathrm{M}$ of AtD14 or OsD14 (Fig 4D), and the presence of up to $100 \mu \mathrm{M}$ of Triton failed to affect the $T_{\text {agg }}$ of AtD14 and OsD14, ruling out strong association with these proteins (Fig 2C). Consistent with these results, application of Triton at concentrations up to $0.5 \mu \mathrm{M}$ did not impact OsD14 or AtD14 YLG hydrolysis activity. Even at a $5 \mu \mathrm{M}$ concentration, Triton decreased the YLG hydrolysis rate of OsD14 or AtD14 by only 10\% (Fig 2G). Importantly, application of $15 \mu \mathrm{M}$ of Triton, a concentration that inhibited S. hermonthica germination by $50 \%$, did neither inhibit rice seed germination nor affected seedling growth (Fig 4E and Appendix Fig S8).

Structural analysis provided a rationale for the absence of D14 binding. The ligand specificity of HTL/D14/KAI2 proteins is determined by the positioning of helices $\alpha 4$ and $\alpha 5$, which frame the binding pocket entrance, and by the residues that line the binding pocket (Figs 5A and B, and EV2). Although most secondary structure elements of ShHTL7 superimpose closely on those of AtD14 and OsD14 (RMSD of $1.12 \AA$ ), the $\alpha 4-\alpha 5$ aperture of ShHTL7 is widened by $\sim 1.5 \AA$ in its $\mathrm{I} 222$ apo form and by $\sim 3 \AA$ in its Triton-bound form through an outward movement of $\alpha 4$ (Fig 5A and B). This $\alpha 4$ opening appears precluded in non-Striga orthologues by the substitution of ShHTL7 L143 with phenylalanine in the $\alpha 4-\alpha 7$ interface (Figs $1 \mathrm{C}$ and E, and EV2). This substitution is homologous and stereochemically equivalent to the L143Y substitution seen in ShHTL5, for which the crystal structure also shows a more inward positioning of $\alpha 4$ (Figs $1 \mathrm{E}$ and $5 \mathrm{~A}$ and $\mathrm{C}$ ). In agreement, Triton failed to bind the ShHTL7 L143Y mutant in vitro and did not inhibit its hydrolysis activity or ShMAX2 binding (Figs 2G and $4 \mathrm{~B}$ and D). Additionally, the substitution of several side chains in the ShHTL7 binding pocket with bulkier ones in non-Striga D14/ KAI2 (L153W, T157F/Y, C194F; Figs 1E and 5B) reduces the size of the binding pocket more than is tolerable by Triton (Fig 5B). Conservation of the active site in D14/KAI2 proteins (Figs 1E and EV2) supports generalization of these results. 


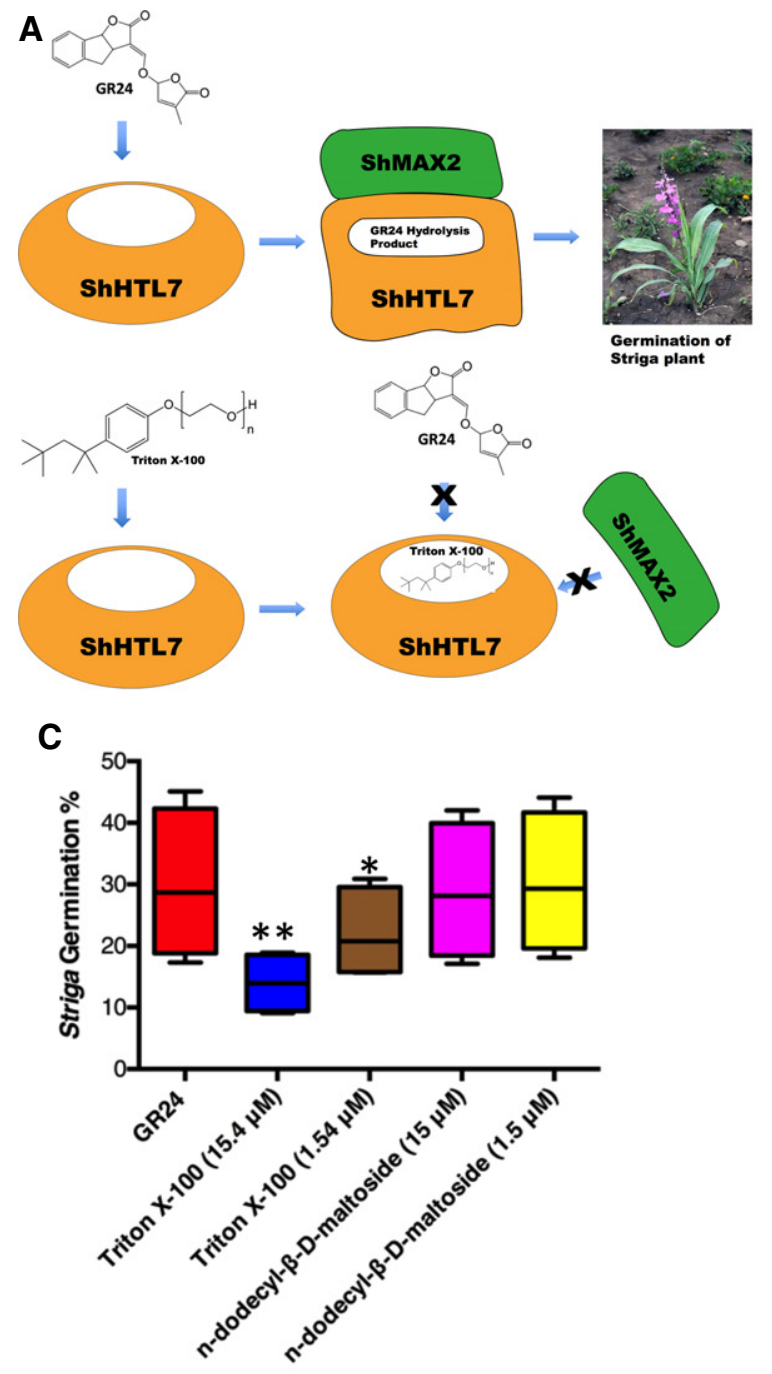

B

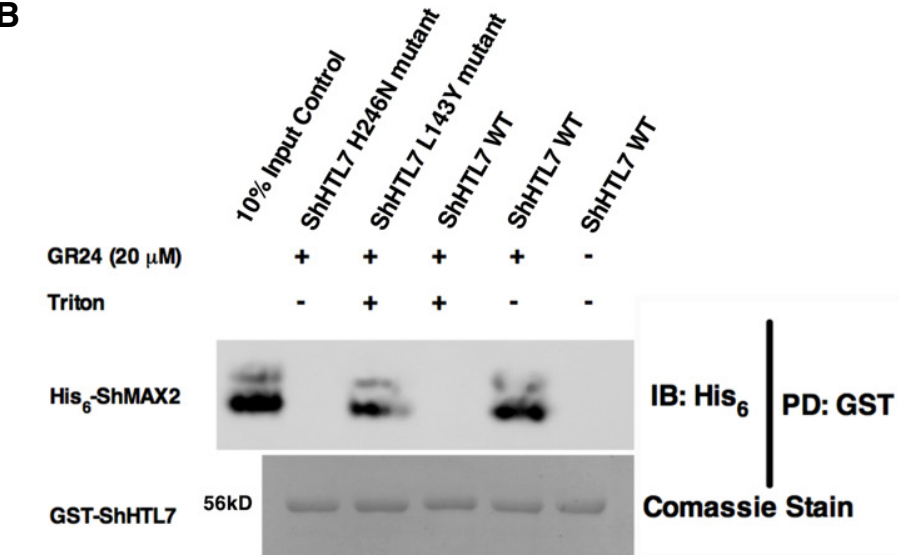

D
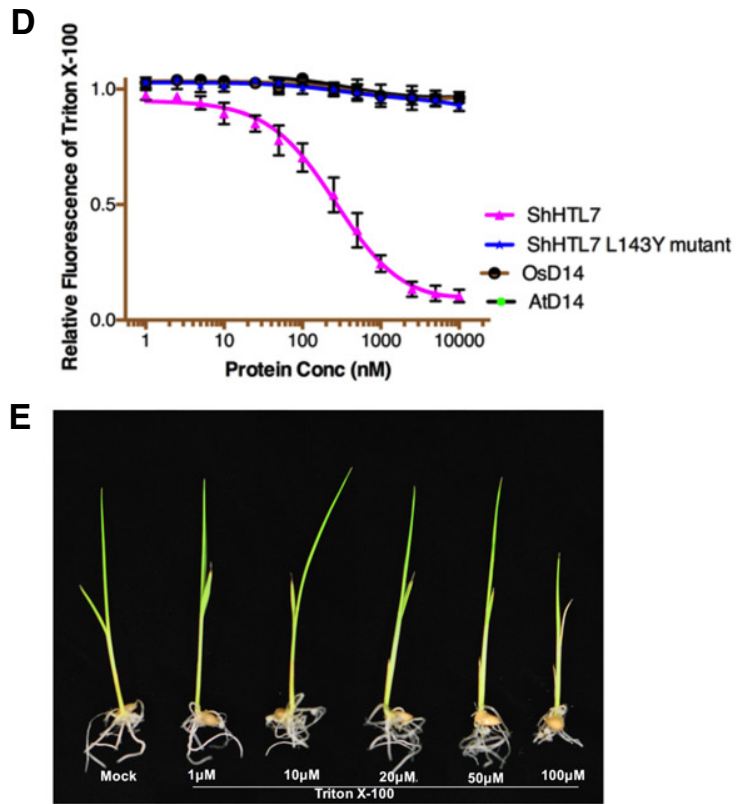

Figure 4. In vitro and in vivo efficacy of Triton in combating activity of ShHTL7 and Striga.

A Schematic diagram depicting the mode of inhibition of ShHTL7 by Triton via blocking the binding pocket of ShHTL7 and thus prevents the access for its substrate Strigolactones such as GR24.

B GST pulldown of His ${ }_{6}$-ShMAX2 with GST-ShHTL7 in the presence of Triton and GR24. The ShHTL7 mutations L143Y and H246N block binding to Triton and GR24, respectively. Ten percent of used ShMAX2 are shown as input control. Full gels are shown in Appendix Fig S5C.

C Percentage of Striga hermonthica seeds germinating after 2 days of treatment with Triton at $15.4(0.001 \%)$ and $1.54 \mu \mathrm{M}(0.0001 \%)$ concentrations and n-dodecyl- $\beta$ D-maltoside at 15 and $1.5 \mu \mathrm{M}$ concentrations in the presence of GR24 at 1, 0.5, 0.25, and $0.125 \mathrm{nM}$ concentrations, respectively. Data are shown as box and whisker plots, where horizontal central value represents median, upper and lower quartiles are the ends of the box, the box spans the interquartile range and whiskers are the maximum and minimum values, $n=6$ (50-100 seeds per replicate), ${ }^{*} P<0.05$, ${ }^{* *} P<0.01$ (one-way ANOVA).

D Intrinsic Triton fluorescence in the presence of increasing concentrations of ShHTL7 and homologs. Data are mean \pm SD, $n=3$.

E Triton effects on Nipponbare rice seedling growth. Representative 2-week-old Nipponbare rice seedlings treated with Triton or water (mock).

\section{Triton-like structures as lead compounds for specific inhibition of Striga seed germination}

Our crystal structures showed that the Triton polyethylene oxide chain also contributes to the binding to ShHTL7. To assess the impact of this chain, we tested commercially available Triton-like compounds with ethylene oxide group sizes from 1 to 40 units (Triton X-100 has 9-10 units; Fig 6A-F). We also included 2-[4-(2,4,4trimethylpentan-2-yl)phenoxy]acetic acid (PAA). Although it has the same hydrophobic tail as Triton, PAA is not a detergent because it carries a phenoxy acid group instead of the polyethylene repeats. All Triton-like compounds inhibited YLG hydrolysis with $\mathrm{IC}_{50}$ values of $1-12 \mu \mathrm{M}$ that were higher, but still in the same range as the one for Triton $(0.47 \mu \mathrm{M}$; Fig $6 \mathrm{G})$. PAA showed the strongest inhibitory effect on ShHTL7 activity in YLG hydrolysis assays ( IC $_{50}$ of $1.48 \mu \mathrm{M}$; Fig $6 \mathrm{E}$ and $\mathrm{G}$ ). Moreover, PAA showed direct binding to ShHTL7 in MST experiments, with a $K_{\mathrm{d}}$ of $1.7 \pm 0.24 \mu \mathrm{M}$, and stabilized ShHTL7 $T_{\text {agg }}$ with the addition of PAA (Fig 7A and B). $10 \mu \mathrm{M}$ PAA also inhibited $S$. hermonthica germination at ratios up to $40 \%$ (Fig 7C and Appendix Fig S8), further establishing that the inhibitory 
A

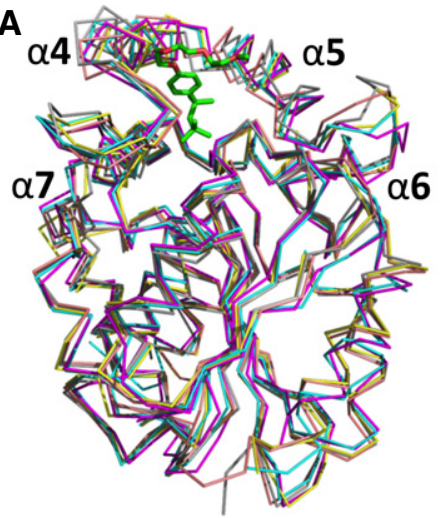

B

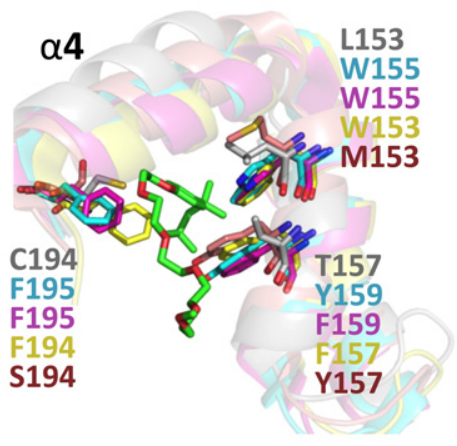

C

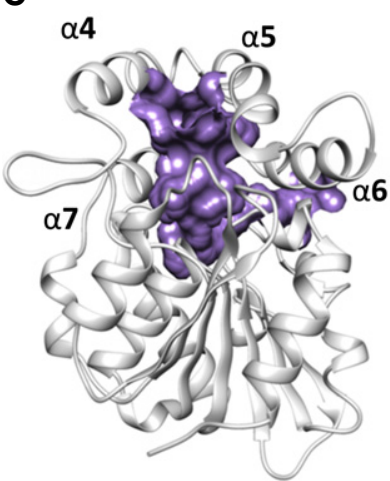

ShHTL7

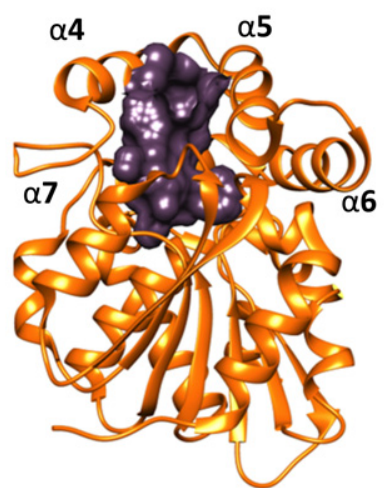

ShHTL5

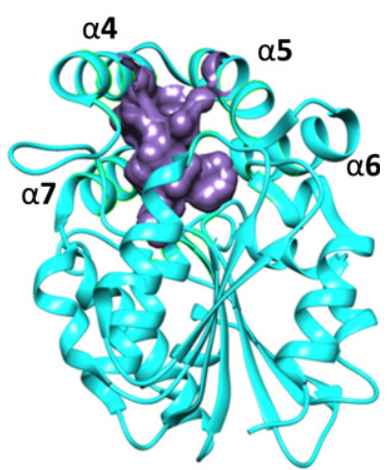

OsD14

Figure 5. Structural determinants of Triton specificity.

A Superimposition of ShHTL7-Triton (space group P65; gray; Triton: green carbons and red oxygens), ShHTL5 (PDB 5CBK; salmon), OsD14 (PDB 4IHA; cyan), AtD14 (PDB 4IH4; magenta) and AtHTL (PDB 4HRY; yellow). The RMSD between structures is $1.12 \AA$.

B Zoom onto helices $\alpha 4$ and $\alpha 5$. Triton and relevant side chains are shown.

C ShHTL7 (gray), ShHTL5 (orange), and OsD14 (cyan) with their ligand-binding pockets shown in surface view.

activity of Triton on SL hydrolysis and Striga seed germination is not simply a non-specific detergent effect. These results showed that very different head groups can achieve similar inhibitory effects in vitro and in planta, suggesting that a larger screen of this moiety may result in compounds with improved efficacy and bioavailability.

\section{Discussion}

The witchweed has become a major threat for food security in subSaharan Africa, and an efficacious herbicide to control this pandemic plant pest is still lacking. Collectively, our data support that Triton-like compounds are a very promising basis for the development of specific inhibitors of Striga germination.

Among ShHTL family members, ShHTL7 stands out as the receptor that binds in vitro to all SLs tested with high affinity [21]. Our analysis now suggests that the broad specificity of ShHTL7 toward SLs is explained by the large volume of the SL binding pocket $\left(1,174.3 \AA^{3}\right.$ compared to 894.5 and $710.1 \AA^{3}$ in ShHTL5 and rice D14, respectively) that can accommodate diverse SLs without steric clashes (Figs 5C and 7D). The increased pocket size is caused by two mechanisms: Firstly, the increased opening between helices 4 and 5, and secondly the replacement of bulky aromatic hydrophobic side chains (F, W, Y) with smaller ones (L, $\mathrm{V}, \mathrm{S}, \mathrm{T})$. This second mechanism is already apparent when comparing D14 with ShHTL5; however, it is further reinforced in ShHTL7, in particular through the presence of a threonine in position 157 instead of a tyrosine (Figs $1 \mathrm{E}$ and 7D). Compared to ShHTL5, ShHTL7 has also substituted four of the flexible methionine residues with more rigid amino acids (L and S; Fig 1E). The resulting reduction in flexibility inside the pocket is expected to reduce the entropic penalty of binding to SLs and hence to generally increase SL affinity. Using transgenic Arabidopsis lines, it was previously shown that ShHTL7 is the most sensitive SL receptor in Striga [22]. Our observation that the ShHTL7-specific inhibition by Triton reduced germination by up to $50 \%$ provides the first direct evidence for the primary role of this receptor in Striga seed germination.

The site used by MAX2 (D3) to bind to AtD14 is 79\% identical in ShMAX2 (Appendix Fig S4), suggesting that SL-bound ShHTLs also need to undergo the same structural rearrangement of helices $\alpha 4$, $\alpha 5$, and $\alpha 6$ for ShMAX2 association as AtD14 in its complex with AtMAX2. Our structural and biophysical analyses demonstrate that GR24 binding leads to loss of helical content, destabilization, and structural changes in ShHTL7, as needed to associate with ShMAX2. In both of our ShHTL7 crystal lattices, $\alpha 4$ and $\alpha 5$ are more distant 
A
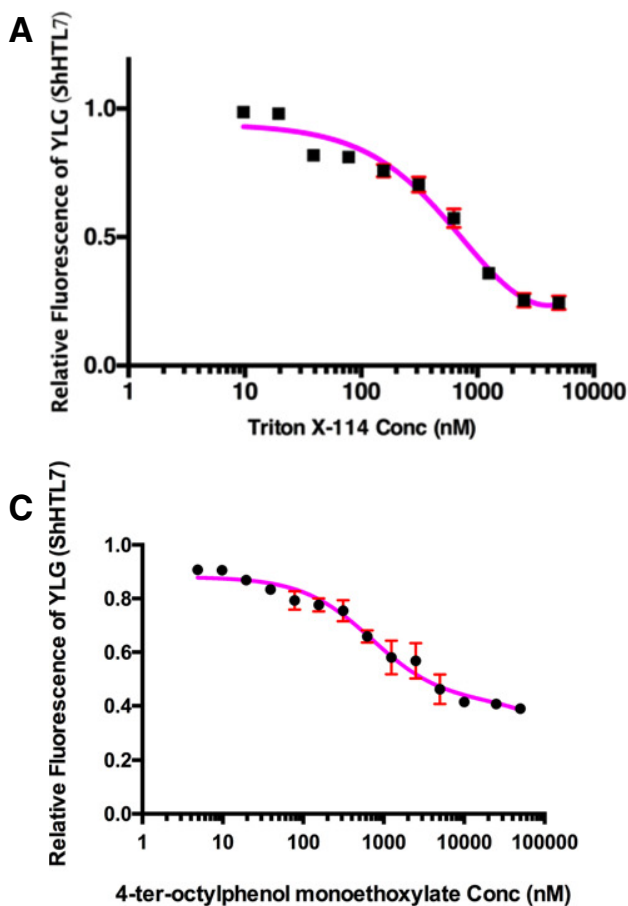

E

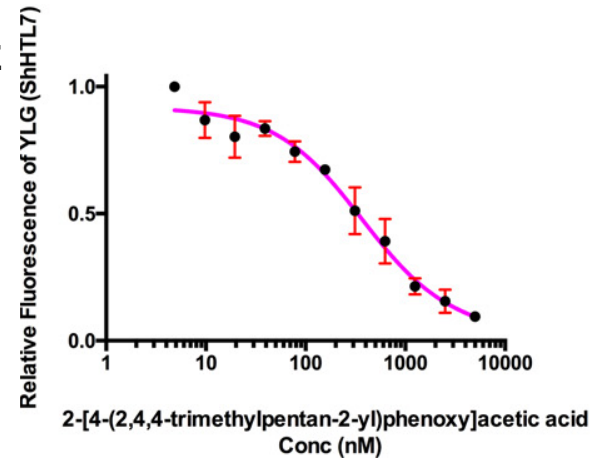

B

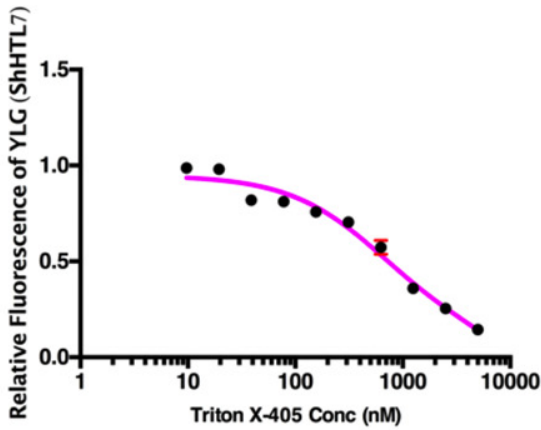

D

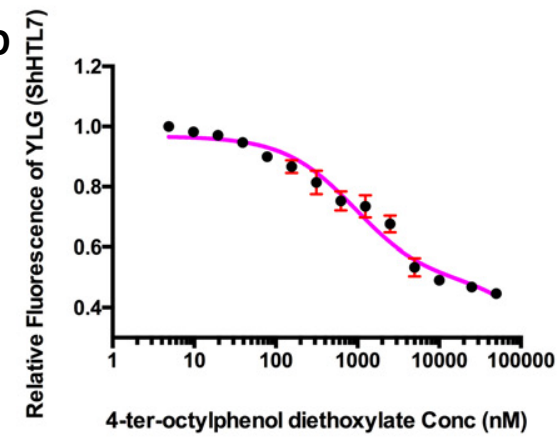

$\mathbf{F}$

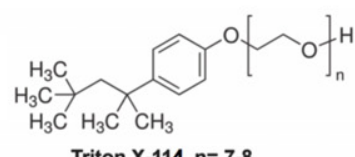

Triton $X-114, n=7-8$

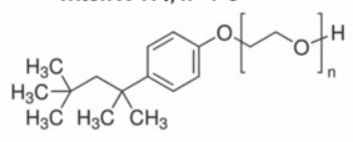

Triton $\mathrm{X}-\mathbf{4 0 5}, \mathrm{n}=\mathbf{4 0}$

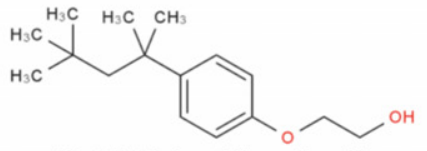

4-tert-Octylphenol Monoethoxylate

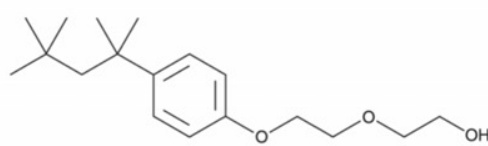

4-tert-Octylphenol Diethoxylate

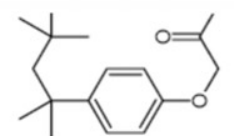

2-[4-(2,4,4-trimethylpentan-2-yl) phenoxy]acetic acid

G

\begin{tabular}{|l|l|l|}
\hline $\begin{array}{l}\text { No. of ethylene } \\
\text { oxide Groups }\end{array}$ & Compound & $I C_{50}(\mu \mathrm{M})$ \\
\hline 40 & Triton X-405 & $11.52 \pm 2.76$ \\
\hline $9-10$ & Triton X-100 & $0.47 \pm 0.11$ \\
\hline $7-8$ & Triton X-114 & $2.15 \pm 0.49$ \\
\hline 2 & 4-ter-octylphenol diethoxylate & $7.48 \pm 2.13$ \\
\hline 1 & 4-ter-octylphenol Monoethoxylate & $9.24 \pm 3.17$ \\
\hline $\begin{array}{l}\text { None (Phenoxy } \\
\text { acetic acid) }\end{array}$ & $\begin{array}{l}\text { 2-[4-(2,4,4-trimethylpentan-2- } \\
\text { yl)phenoxy]acetic acid }\end{array}$ & $1.48 \pm 0.41$ \\
\hline
\end{tabular}

Figure 6. Hydrolysis activity of ShHTL7 in the presence of Triton X-100-like compounds.

A-E Triton X-114 (A), Triton X-405 (B), 4-ter-octylphenol monoethoxylate (C), 4-ter-octylphenol diethoxylate (D), 2-[4-(2,4,4-trimethylpentan-2-yl)phenoxy]acetic acid (E). Data are mean $\pm \mathrm{SD}, n=3$.

$\mathrm{F} \quad$ Chemical structures of the tested compounds.

G $\quad I_{50}$ values for Triton-like compounds based on ShHTL7 YLG hydrolysis assays.

from the underlying helices $\alpha 7$ and $\alpha 6$ than in D14 or ShHTL5. $\alpha 4$ and $\alpha 5$ are also anchored with smaller side chains (Fig 7E and F). Combined, the loser attachment and shorter side chains of this region may facilitate its restructuring upon binding to ShMAX2, which may contribute to the high capacity of ShHTL7 to trigger downstream signaling.

Interestingly, the picomolar sensitivity of ShHTLs in planta does not correlate with their affinity for SLs in vitro, inferring that other factors contribute $[21,22]$. The discrepancy between in vitro and in planta measurements is in line with previous observations. Toh et al reported that picomolar concentrations of GR24 are sufficient to trigger seed germination in transgenic Arabidopsis lines expressing ShHTL7, whereas the in vitro YLG assay with recombinant ShHTL6 and ShHTL7 revealed an IC $_{50}$ of $\sim 0.5 \mu \mathrm{M}$ for GR24 $[21,22]$. Thus, in vitro binding or hydrolysis assays, GR24-based seed germination activity in transgenic Arabidopsis, and Striga germination through natural SLs in the field cannot be compared. The apparent SL sensitivity in planta may be strongly influenced by processes such as bioavailability, local SL concentration, or interactions with other proteins. Hence, the inhibitory effect of Triton in planta might be limited by its bioavailability and ability to be uptaken and transported within Striga seeds.

Chemical control of Striga is currently limited due to the unavailability of compounds that specifically target Striga, without affecting the host plants. Here, we identified Triton X-100 as a specific inhibitor of the most sensitive Striga SL receptor, ShHTL7. The structural match, the high in vitro affinity, and the robust in vivo activity identify Triton as a promising lead compound for the design of Striga-specific herbicides. We showed that Triton X-100 derived structures with different head groups also inhibited ShHTL7, opening up possibilities of designing a new class of Striga inhibitors. Moreover, the Triton-ShHTL7 complex structure suggests that 
A
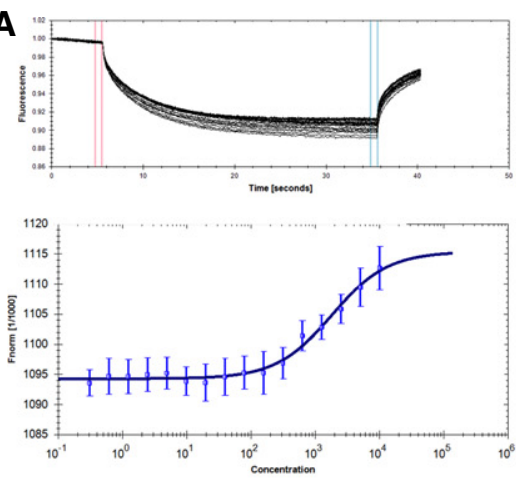

ShHTL7 vs PAA

D

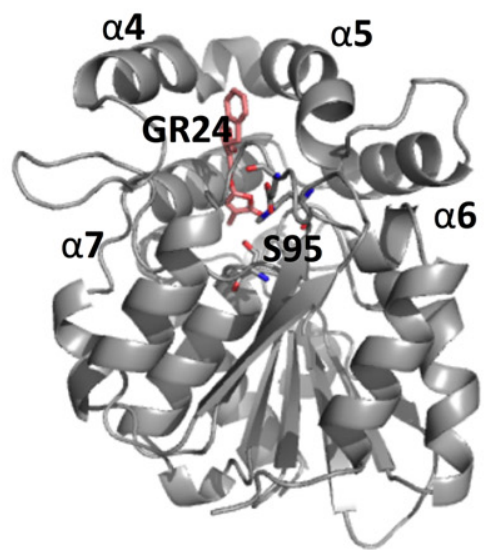

B

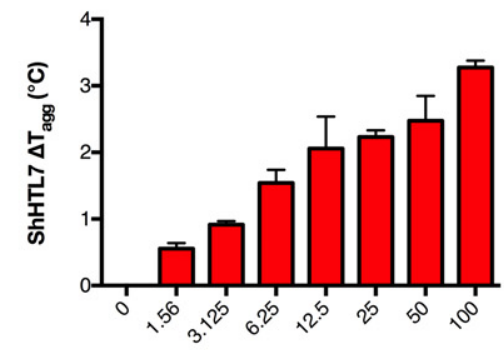

2-[4-(2,4,4-trimethylpentan-2-yl)phenoxy]acetic acid (PAA) Conc $(\mu \mathrm{M})$
C

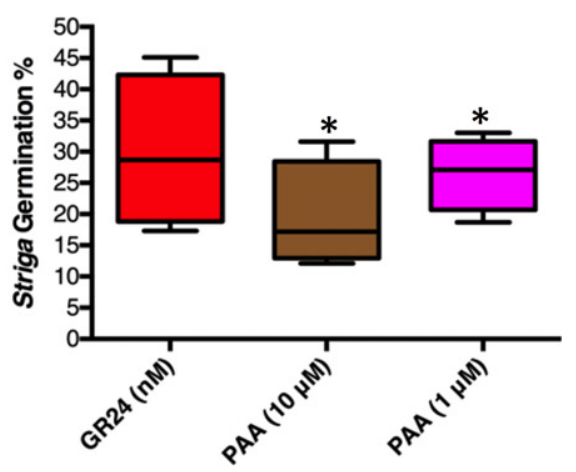

E

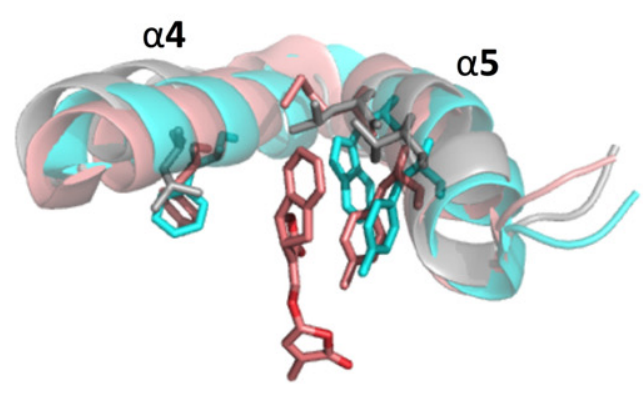

$\mathbf{F}$

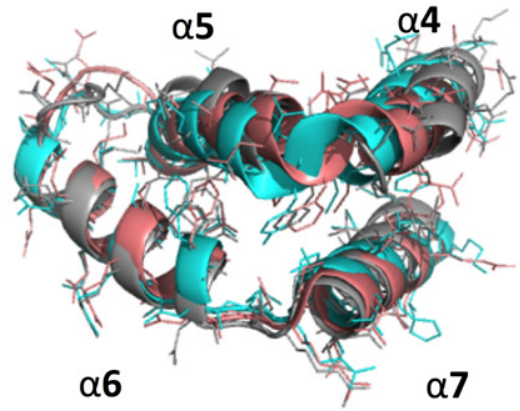

GR24

Figure 7. PAA binds to ShHTL7 and inhibits Striga germination and the structural requirement for ShHTL7 to accommodate its substrate.

A Thermophoresis data of fluorescently labeled ShHTL7 binding to different concentrations of PAA from $25 \mu \mathrm{M}$ to sub-nanomolar range. Data are mean \pm SD of the fluorescence counts from the labeled protein, $n=3$.

B The change in ShHTL7 aggregation temperature $\left(\Delta T_{\text {agg }}\right)$ plotted against PAA concentrations. Data are mean $\pm S D, n=3$.

C Percentage of Striga hermonthica seeds germinating after 2 days of treatment with 2-[4-(2,4,4-trimethylpentan-2-yl)phenoxy]acetic acid at 10 and $1 \mu \mathrm{M}$ in the presence of GR24 at 1, 0.5, 0.25, and $0.125 \mathrm{nM}$ concentrations, respectively. Data are shown as box and whisker plots, where horizontal central value represents median, upper and lower quartiles are the ends of the box, the box spans the interquartile range and whiskers are the maximum and minimum values, $n=6$ ( $50-$ 100 seeds per replicate), ${ }^{*} P<0.05$ (one-way ANOVA).

D ShHTL7 (space group 1222; gray) superimposed with GR24 (orange stick model) transposed from its position bound to OsD14 (PDB 5DJ5).

E Superimposition of helices $\alpha 4$ and $\alpha 5$ of ShHTL7 (gray), ShHTL5 (PDB 5CBK; salmon), OsD14 (PDB 5DJ5; cyan). The position of GR24 (bound to OsD14) and relevant side chains are shown.

F Superimposition of helices $\alpha 4, \alpha 5, \alpha 6$, and $\alpha 7$ ShHTL7 (gray), ShHTL5 (PDB 5CBK; salmon), and OsD14 (PDB 5DJ5; cyan) and the side chains are shown as lines.

derived compounds with a hydrophobic moiety that fills the gap between Triton and the active site pocket may exhibit higher inhibitory activity. A further improvement might be achieved by designing derivatives that reach and covalently bind to the active site.

Its potency, persistence, and entire filling of the ShHTL7 active site distinguish Triton from compounds such as glycerol and PMSF, which are frequently found attached non-specifically to small regions of protein active sites. Moreover, the fact that the action of Triton can be recapitulated by the non-detergent Triton-like compound PAA, but not by the detergent $\mathrm{nDM}$, excludes that our observations are simply caused by non-specific detergent effects. We show that the occupation of the ShHTL7 binding pocket by Triton precludes the structural changes required for downstream signaling through ShMAX2. The high resolution of our structural data delivers the atomic details required for the rational development of inhibitors with improved efficacy. Indeed, our structural analysis showed that the binding pocket of ShHTL7 is too different to be modeled correctly based on known homologous structures.

Our in vivo assessment of Striga germination inhibition by Triton showed robust effects, with, however, noticeable differences between seed batches and assay conditions. Based on structural modeling of all ShHTL7 family members, the reduction of germination by Triton is predicted to be caused only by inhibition of ShHTL7. In transgenic $A$. thaliana, ShHTL4-6 showed EC 50 values of $0.01-0.3 \mu \mathrm{M}$ for GR24 [21]. These concentrations are far above the picomolar sensitivity of Striga seeds for SLs [28], and hence, the contributions of ShHTL family members other than ShHTL7 are expected to be of only minor importance in planta. 
Interestingly, it was earlier noted, but not further investigated, that Triton X-100 concentrations above $0.001 \%$ hindered seed germination of Orobanche cumana and Orobanche minor [29], suggesting that this compound might also be used to combat root parasitic weeds other than S. hermonthica.

In conclusion, our work confirms and explains the primordial role of ShHTL7 as SL receptor in Striga. Our analysis paves the way for realizing a promising approach in combating Striga, which is based on applying Striga-specific germination inhibitors in the presence of host crops throughout the planting season. Thus, such Triton-derived inhibitors can complement or replace the suicidal germination approach and provide a highly needed addition to the insufficient tool-box of anti-Striga measures.

\section{Materials and Methods}

\section{Cloning, expression and protein purification}

Striga hermonthica ShHTL7 cDNA (GenBank accession: KR013127) was kindly provided by Prof. Tadao Asami (The University of Tokyo, Japan). ShHTL7s95C mutant, A. thaliana D14 (AtD14) cDNA (GenBank accession: AY097402), and Oryza sativa D14 (OsD14) cDNA (GenBank accession: BAF11220) were synthesized and cloned

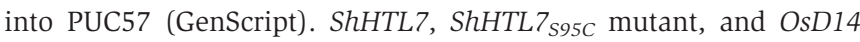
were amplified by PCR using primers [30] (Appendix Table S1). The obtained fragments were digested with BamHI and XhoI and ligated into accordingly treated pGEX-6P-1 expression vector (GE Healthcare). AtD14 was amplified by PCR using AtD14-F/AtD14-R primers (Appendix Table S1), and the obtained fragment was digested with EcoRI and SalI, and ligated into EcoRI/SalI digested pGEX-6P-1 expression vector. ShHTL7-L143Y mutant was generated by PCR according to [30] and using ShHTL7-L143Y-F/ShHTL7-L143Y-R primers (Appendix Table S1) designed to introduce the desired mutation in the pGEX-6P-1-ShHTL7 plasmid. After PCR, the mixture was digested with DpnI and transformed into Escherichia coli TOP10 cells, to generate the plasmid with the introduced mutation. All plasmids were verified by sequencing (KAUST Bioscience Core Lab). The plasmids were then transformed into E. coli BL21 (DE3) cells. Cells were grown in LB broth containing ampicillin $(100 \mathrm{mg} / \mathrm{ml})$ at $37^{\circ} \mathrm{C}$ until an $\mathrm{OD}_{600}$ of 0.6 and for NMR ${ }^{15} \mathrm{~N}$ labeling, and the cells were grown in M9 minimal media supplemented with ${ }^{15} \mathrm{~N}$ ammonium chloride. Expression was induced by adding $150 \mu \mathrm{M}$ isopropyl $\beta$-D-1-thiogalactopyranoside (IPTG), and cultures were incubated at $16^{\circ} \mathrm{C}$ overnight under shaking. Cells were harvested by centrifugation at $8,000 \mathrm{~g}$ for $10 \mathrm{~min}$. Cell pellet of one liter culture was resuspended in $25 \mathrm{ml}$ lysis buffer ( $50 \mathrm{mM}$ Tris- $\mathrm{HCl}$ (pH 8.0), $200 \mathrm{mM}$ $\mathrm{NaCl}, 3 \mathrm{mM}$ DTT, $700 \mu \mathrm{M}$ Triton X-100, or $9.6 \mathrm{mM}$ Tween-20) and lysed by sonication. Cell debris was removed by centrifugation at $30,000 \mathrm{~g}$ for $30 \mathrm{~min}$, and proteins were purified from the obtained supernatant using Glutathione Sepharose 4B resins (GE Healthcare). The N-terminal GST tag of ShHTL7 was removed by overnight incubation with PreScission Protease (GE Healthcare) at $4^{\circ} \mathrm{C}$. After GST cleavage, protein was further purified on a HiLoad16/60 Superdex 200 prep-grade gel filtration column (GE Healthcare) using a buffer containing $10 \mathrm{mM}$ HEPES ( $\mathrm{pH} 7.5$ ), $50 \mathrm{mM} \mathrm{KCl}, 3 \mathrm{mM}$ DTT. The purified protein was concentrated to $10 \mathrm{mg} / \mathrm{ml}$ and stored at $-80^{\circ} \mathrm{C}$. The expression and purification protocol for ShHTL7 was also used for AtD14 and OsD14 proteins, except that AtD14 and OsD14 were eluted from glutathione resins using $0.2 \mathrm{mM}$ reduced glutathione (Sigma) without cutting the GST tag. Eluted proteins were passed through gel filtration equilibrated with buffer containing $10 \mathrm{mM}$ HEPES ( $\mathrm{pH}$ 7.5), $50 \mathrm{mM} \mathrm{KCl,} 3 \mathrm{mM}$ DTT and further used for in vitro assays.

\section{Crystallization and structure determination}

The hanging drop vapor diffusion method was used for crystallization. Initial plate crystals were obtained for apo-ShHTL7 and ShHTL7 co-crystallized with $1 \mathrm{mM}$ rac-GR24 (Chiralix) by equilibrating $1.0 \mu \mathrm{l}$ of protein $(15 \mathrm{mg} / \mathrm{ml})$ mixed with $1.0 \mu \mathrm{l}$ of reservoir

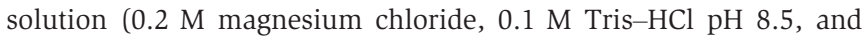
$30 \%$ PEG 4000). These crystals were crushed and used as microseeds to obtain diffraction-quality crystals under same crystallization conditions. For ShHTL7 and the ShHTL7 $7_{\text {S95C }}$ mutant with Triton X-100, diffraction-quality crystals were obtained by equilibrating $1.0 \mu \mathrm{l}$ of protein $(10 \mathrm{mg} / \mathrm{ml})$ mixed with $1.0 \mu \mathrm{l}$ of reservoir solution (1 M lithium sulfate, 0.1 M HEPES pH 7.5), in addition apo ShHTL7 crystallized in reservoir solution containing $0.1 \mathrm{M} \mathrm{MES} \mathrm{pH}$ 6.5 and $1 \mathrm{M}$ magnesium sulfate. All crystals grew in 1-3 days at $23^{\circ} \mathrm{C}$. For data collection, $25 \%$ glycerol was added to the well solution as a cryo-protectant, and the crystals were flash-cooled in liquid nitrogen. All data for apo ShHTL7, ShHTL7-Triton X-100, and

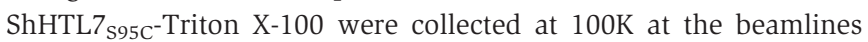
Proxima 1 and Proxima $2 \mathrm{~A}$ at the SOLEIL Synchrotron (France), using a Pilatus $6 \mathrm{M}$ and EIGER $6 \mathrm{M}$ detector, respectively (proposal numbers 20160098, 20161236, 20170193). The data were processed in XDS. The crystal structure of ShHTL7 ${ }_{\mathrm{S} 95 \mathrm{C}}$-Triton X-100 was determined by molecular replacement using MoRDa with the ShHTL5 structure (PDB 5CBK) as search model. The refined ShHTL7 ${ }_{\text {S95C }}$ model was then used as MR template for ShHTL7-Triton X-100 and apo ShHTL7. The structures were manually inspected using Coot and refined using Phenix Refine (Table 1). The figures were drawn using PYMOL. The active site pocket volume was calculated following the previously published method [22].

\section{Yoshimulactone green (YLG) hydrolysis assays}

In vitro YLG hydrolysis assays were performed using $3 \mu \mathrm{M}$ of ShHTL7, ShHTL7-L143Y, AtD14, and OsD14 in a reaction buffer (1× PBS) with $0.1 \%$ dimethyl sulfoxide (DMSO) in a $100 \mu$ l volume on a 96-well black plate (Greiner). The fluorescent intensity was measured by SpectraMax i3 (Molecular Devices) using an excitation wavelength of $480 \mathrm{~nm}$ and a detection wavelength of $520 \mathrm{~nm}$. ShHTL7, ShHTL7-L143Y, AtD14, and OsD14 were mixed with Triton $\mathrm{X}-100$, using serial dilutions to cover the Triton X-100 concentration range from $5 \mu \mathrm{M}$ until $4.87 \mathrm{nM}$. Protein-Triton mixes were pre-incubated for 30 min. $1 \mu \mathrm{M}$ YLG (Tokyo Chemical Industry Co. Ltd.) was then added, and the assay was incubated for $60 \mathrm{~min}$. The change in fluorescence observed over the course of $1 \mathrm{~h}$ incubation of YLG in buffer without protein was subtracted from the data collected in the presence of protein. The resulting relative fluorescence values were analyzed. The inhibitory curves and $\mathrm{IC}_{50}$ values were plotted using GraphPad (Prism 6) four-parameter logistic curve. The rate of YLG hydrolysis by ShHTL7 was recorded following the above method at 10-min interval for $2 \mathrm{~h}$. 


\section{Triton X-100 fluorescence}

Triton X-100 has an excitation and emission wavelength of 275 and 302 nm, respectively. ShHTL7, ShHTL7-L143Y, AtD14, and OsD14 were incubated in $50 \mathrm{mM}$ Tris- $\mathrm{HCl} \mathrm{pH} 7.5,150 \mathrm{mM} \mathrm{NaCl}$, with or without $0.005 \%$ Tween-20, at concentrations ranging from $10 \mu \mathrm{M}$ to $1 \mathrm{nM}$ (obtained using serial dilutions) with $154 \mathrm{nM}$ of Triton X-100 for $1-2 \mathrm{~h}$, and fluorescence values were then measured by SpectraMax i3 (Molecular Devices) at excitation and detection wavelengths of 275 and $302 \mathrm{~nm}$, respectively. The fluorescence exhibited by Triton X-100 alone was subtracted from the fluorescence in the presence of protein. Data were normalized to the background fluorescence from the protein to obtain the change in Triton X-100 fluorescence intensity. To determine the $K_{\mathrm{d}}$, the changes in Triton X-100 fluorescence intensity were plotted against the concentration of the protein using the single binding site model implemented in GraphPad (Prism 6).

\section{Differential static light scattering (DSLS)}

The specific aggregation temperature, $T_{\text {agg, }}$ at which ShHTL7, ShHTL7 $_{\mathrm{L} 143 \mathrm{Y}}$, AtD14, and OsD14 aggregated in the presence or absence of Triton X-100, GR24, and PAA was measured using DSLS on the Stargazer system (Harbinger Biotechnology and Engineering Corporation, Markham, Canada). Protein samples at a concentration of $10 \mu \mathrm{M}$ were overlaid with mineral oil in a clear bottom 384-well black plate (Corning) and heated from 20 to $95^{\circ} \mathrm{C}$ at $1^{\circ} \mathrm{C} / \mathrm{min}$; light scattering was detected by a CCD camera every $0.5^{\circ} \mathrm{C}$ in the presence or absence of varying concentrations of Triton X-100, GR24, and PAA yielding the difference in aggregation temperature at a particular concentration from apoproteins $\left(\Delta T_{\text {agg }}\right)$. Data were normalized, and $\Delta T_{\text {agg }}$ plotted against the Triton X-100, GR24, and PAA concentration. Resulting data were plotted to determine the change in $T_{\text {agg }}$ of all proteins when bound to Triton X-100, GR24, and PAA using GraphPad (Prism 6).

\section{Mass spectrometry (MS) for molecular weight determination}

Mass spectrometric analysis of ShHTL7-apo, ShHTL7 $7_{\mathrm{S} 95 \mathrm{C}}$, and ShHTL7 bound to Triton X-100 was performed using electrospray ionisation system Q-ToF (Bruker Maxis) equipped with liquid chromatography (Agilent Technologies). Under denaturing conditions, the protein was desalted using C4 Ziptip (EMD Millipore) and eluted into $50 \%$ acetonitrile/ $50 \%$ water $/ 0.1 \%$ formic acid. For each analysis, a 3-6 $\mu \mathrm{l}$ aliquot of the protein solution was loaded. Mass spectra were processed by inbuilt software for Bruker maxis.

\section{Striga hermonthica germination bioassays}

Striga hermonthica seeds were collected from Sorghum bicolor field in Sudan. For germination bioassays, Striga seeds were first sterilized and pre-conditioned as described before [31]. The pre-conditioned Striga seeds were treated with Triton X-100 concentrations 1.54 and $15.4 \mu \mathrm{M}$ along with GR24 (final concentration of 1.0, 0.5, 0.25 , and $0.125 \mathrm{nM}$ ). Triton X-100/GR24 mixtures were applied (50 $\mu$ l per disk) in four replicates. Corresponding volumes of GR24 and sterile MiliQ water were included as positive and negative control, respectively. The petri dishes were sealed with parafilm, enfolded with aluminum foil, and incubated for $48 \mathrm{~h}$ at $30^{\circ} \mathrm{C}$. The germinated (seeds with radicle emerging through the seed coat) and non-germinated Striga seeds were recorded under a binocular, and germination percentage was calculated. For germination assays against n-dodecyl- $\beta$-D-maltoside, it was done at $15,1.5 \mu \mathrm{M}$ and for PAA it was $10,1 \mu \mathrm{M}$, respectively, and same method as above was adopted for the assays with same GR24 concentrations.

\section{Rice seeds germination assays and growth of seedlings}

The rice seeds $c v$. Nipponbare were surface-sterilized using $50 \mathrm{ml}$ (2.5\%) sodium hypochlorite with $0.4 \%$ of Tween-20 for $15 \mathrm{~min}$. After six subsequent washing steps with sterilized water, the rice seeds were kept in water at $30^{\circ} \mathrm{C}$ for overnight imbibition. About $2 \mathrm{ml}$ of Triton X-100 concentrations ranging from 1 to $100 \mu \mathrm{M}$ and sterile MilliQ water (control) was added in 24-well plates with six replications. One sterilized rice seed was put in each well and allowed to germinate for $48 \mathrm{~h}$ at $30^{\circ} \mathrm{C}$. The germinated (radicle emergence) and non-germinated seeds were recorded, and germination percentage was calculated. Then, same seeds were allowed to grow under light in growth cabinet for another 1 week. The length of 2 -week-old seedlings was measured with roller and averaged.

\section{GST pulldown of ShMAX2}

Purified GST-ShHTL7 wt, ShHTL7 L143Y, and ShHTL7-H246N mutants were bound to the Glutathione Sepharose 4B Beads, where Triton is used in the lysis step and in certain samples Tween-20 was used instead of Triton. Bound proteins were incubated with $20 \mu \mathrm{M}$ GR24 and His $_{6}$-ShMAX2 lysate for $2 \mathrm{~h}$ and then washed with lysis buffer for three times and then ShHTL7 wt and mutants were eluted with the addition of $20 \mathrm{mM}$ reduced glutathione. Thus, eluted protein was subjected to Western blot using antibody specific to 6XHis (anti-His).

\section{Ligand-binding and stabilization of ShHTL7 by chemical shift perturbation}

To determine ShHTL7 ligand binding and stabilization by NMR, the chemical shift changes induced in ${ }^{15} \mathrm{~N}$ HSQC spectra of ShHTL7 by the presence of GR24 and simultaneously in the presence of Triton X-100 was recorded. All spectra were recorded at $298 \mathrm{~K}$ on a Bruker Avance $950 \mathrm{MHz}$ spectrometer equipped with TCI cryoprobe. Single-labeled ${ }^{15} \mathrm{~N}$ recombinant ShHTL7 $(0.3 \mathrm{mM})$ was titrated by addition $0.45 \mathrm{mM}$ unlabeled GR24, and ${ }^{15} \mathrm{~N}$-labeled ShHTL7 $(0.3 \mathrm{mM})$ purified in the presence of Triton X-100 was titrated by addition $0.45 \mathrm{mM}$ unlabeled GR24. The peak shifts were analyzed using Topspin software program, and the data were overlapped to identify the changes in the chemical shift.

\section{Micro scale thermophoresis (MST) for ShHTL7 binding to ligands}

ShHTL7 was N-terminally labeled with RED dye (NT-647) following the protocol from manufacturer (NanoTemper, Germany). Free dye was removed by passing through the Sephadex G-25 column. Serial dilutions of unlabeled Triton X-100, GR24, and PAA ranging from $25 \mu \mathrm{M}$ to sub-nanomolar range were mixed with $20 \mathrm{nM}$ of 
NT-647-labeled ShHTL7 in buffer (50 mM Tris-HCl, $150 \mathrm{mM} \mathrm{NaCl}$, $0.005 \%$ Tween-20) and incubated for $2.5 \mathrm{~h}$ in the studies for Triton X-100 and PAA except with GR24, and it was recorded after 10-min incubation. About $10 \mu \mathrm{l}$ of sample was loaded into premium monolith NT capillaries, and assays were carried out in a NanoTemper Monolith NT.015T. The red fluorescence was excited by a lightemitting diode, and its emission was recorded on the focused part of capillaries. Using laser diode, a microscopic temperature gradient was created at the same location and the fluorescence depletion was measured. $K_{\mathrm{d}}$ values were determined by plotting the concentrations of unlabeled ligands against the changes in fluorescent thermophoresis signal, and the data were analyzed using the NanoTemper analysis software.

\section{Drug affinity response target stability (DARTS) assay}

ShHTL7 and mutants were diluted to a concentration of $30 \mu \mathrm{M}$ in $50 \mathrm{mM}$ Tris- $\mathrm{HCl} \mathrm{pH} 7.5,150 \mathrm{mM} \mathrm{NaCl}, 0.005 \%$ Tween-20, incubated with GR24 at final concentration of $100 \mu \mathrm{M}$ for $5 \mathrm{~min}$, and separately with $100 \mu \mathrm{M}$ Triton X-100 for $2 \mathrm{~h}$. One sample was kept aside for use as an undigested control. Proteinase $\mathrm{K}$ (Hampton Research) was then added to ShHTL7 protein to a final protease concentration of $10 \mu \mathrm{g} / \mathrm{ml}$. Reactions were incubated at room temperature for $5 \mathrm{~min}$ and then SDS-PAGE loading buffer was added to stop the reaction, and the samples were immediately boiled for $3 \mathrm{~min}$. Equal amounts of each sample, corresponding to $10 \mu \mathrm{g}$ of protein, were run on a $4-20 \%$ gradient polyacrylamide gel (Bio-Rad) and analyzed by Coomassie Blue staining [32].

\section{Small-angle X-ray scattering}

Experiments were performed at the SWING beamline (SOLEIL, Saint-Aubin, France), using the Biology Laboratory 1 (proposal number 20170193). The wavelength was set to $\lambda=1.03 \AA$. The distance of the sample to the detector was $1.8 \mathrm{~m}$, resulting in the momentum transfer range of $0.01 \AA-1<q<0.5 \AA-1$. Between 30 and $50 \mu$ of protein was used. For each sample, two concentrations and two buffer blanks were measured. Protein and buffer data were selected and averaged. Buffer data were subtracted from the protein data using SWING's on-site software. Programs of the ATSAS suite [33] were used for data processing, Guinier plots, distance distributions (PRIMUS), model fitting (CRYSOL), and figure preparation (SASPLOT).

\section{Data availability}

The coordinates for the structures reported in this paper have been deposited in PDB under accession numbers 5Z8P, 5Z95, 5Z82, and $5 Z 89$.

Expanded View for this article is available online.

\section{Acknowledgements}

We acknowledge SOLEIL for provision of synchrotron radiation facilities, and we would like to thank L. Chavas, P. Legrand, S. Sirigu, and P. Montaville for assistance in using beamline PROXIMA 1; G. Fox, M. Savko, and B. Shepard for assistance in using beamline PROXIMA 2A; and J. Perez and A. Thureau for assistance in using the beamline SWING. We thank the KAUST
Bioscience Core Labs for their support, and we thank L. Jaremko for help with the NMR analysis. This research was supported by the Bill \& Melinda Gates Foundation (OPP1136424); and the King Abdullah University of Science and Technology (KAUST).

\section{Author contributions}

STA and SA-B conceived the idea for the study. USH and IH designed the experiments. STA collected and analyzed the SAXS data. STA and USH performed the protein structural studies. USH and IH performed the assays for characterization. XG collected and analyzed the NMR data. MJ and BAK did the Striga bioassays and analyzed the data. RAZ and DK helped in the protein studies. STA, SA-B, USH, and IH wrote the manuscript.

\section{Conflict of interest}

The authors declare that they have no conflict of interest.

\section{References}

1. Xie X, Yoneyama K, Yoneyama K (2010) The strigolactone story. Annu Reu Phytopathol 48: 93-117

2. Ejeta G (2007) The Striga scourge in Africa: a growing pandemic. In Inte grating new technologies for Striga control: towards ending the witchhunt, Ejeta G, Gressel J (eds), pp 3-16. Singapore: World Scientific Publishing Co. Pte Ltd

3. Parker C (2012) Parasitic weeds: a world challenge. Weed Sci 60: $269-276$

4. Gomez-Roldan V, Fermas S, Brewer PB, Puech-Pages V, Dun EA, Pillot JP Letisse F, Matusova R, Danoun S, Portais JC et al (2008) Strigolactone inhibition of shoot branching. Nature 455: 189-194

5. Umehara M, Hanada A, Yoshida S, Akiyama K, Arite T, Takeda-Kamiya N, Magome H, Kamiya Y, Shirasu K, Yoneyama K et al (2008) Inhibition of shoot branching by new terpenoid plant hormones. Nature 455: 195-200

6. Alder A, Jamil M, Marzorati M, Bruno M, Vermathen M, Bigler P, Ghisla S, Bouwmeester H, Beyer P, Al-Babili S (2012) The path from beta-carotene to carlactone, a strigolactone-like plant hormone. Science 335: 1348-1351

7. Jia KP, Baz L, Al-Babili S (2018) From carotenoids to strigolactones. J Exp Bot 69: $2189-2204$

8. Al-Babili S, Bouwmeester HJ (2015) Strigolactones, a novel carotenoidderived plant hormone. Annu Reu Plant Biol 66: 161-186

9. Hamiaux C, Drummond RS, Janssen BJ, Ledger SE, Cooney JM, Newcomb RD, Snowden KC (2012) DAD2 is an alpha/beta hydrolase likely to be involved in the perception of the plant branching hormone, strigolactone. Curr Biol 22: 2032-2036

10. Zhao LH, Zhou XE, Wu ZS, Yi W, Xu Y, Li S, Xu TH, Liu Y, Chen RZ, Kovach A et al (2013) Crystal structures of two phytohormone signaltransducing alpha/beta hydrolases: karrikin-signaling KAI2 and strigolactone-signaling DWARF14. Cell Res 23: 436-439

11. Zhao LH, Zhou XE, Yi W, Wu Z, Liu Y, Kang Y, Hou L, de Waal PW, Li S, Jiang $Y$ et al (2015) Destabilization of strigolactone receptor DWARF14 by binding of ligand and E3-ligase signaling effector DWARF3. Cell Res 25: $1219-1236$

12. Yao R, Ming Z, Yan L, Li S, Wang F, Ma S, Yu C, Yang M, Chen L, Chen L et al (2016) DWARF14 is a non-canonical hormone receptor for strigolactone. Nature 536: $469-473$

13. Nakamura H, Xue YL, Miyakawa T, Hou F, Qin HM, Fukui K, Shi X, Ito E, Ito S, Park SH et al (2013) Molecular mechanism of strigolactone perception by DWARF14. Nat Commun 4: 2613 
14. de Saint Germain A, Clave G, Badet-Denisot MA, Pillot JP, Cornu D, Le Caer JP, Burger M, Pelissier F, Retailleau P, Turnbull C et al (2016) An histidine covalent receptor and butenolide complex mediates strigolactone perception. Nat Chem Biol 12: 787-794

15. Zhou F, Lin Q, Zhu L, Ren Y, Zhou K, Shabek N, Wu F, Mao H, Dong W, Gan L et al (2013) D14-SCF(D3)-dependent degradation of D53 regulates strigolactone signalling. Nature 504: $406-410$

16. Jiang L, Liu X, Xiong G, Liu H, Chen F, Wang L, Meng X, Liu G, Yu H, Yuan $Y$ et al (2013) DWARF 53 acts as a repressor of strigolactone signalling in rice. Nature 504: 401-405

17. Waters MT, Nelson DC, Scaffidi A, Flematti GR, Sun YK, Dixon KW, Smith SM (2012) Specialisation within the DWARF14 protein family confers distinct responses to karrikins and strigolactones in Arabidopsis. Development 139: 1285-1295

18. Guo Y, Zheng Z, La Clair JJ, Chory J, Noel JP (2013) Smoke-derived karrikin perception by the alpha/beta-hydrolase KAl2 from Arabidopsis. Proc Natl Acad Sci USA 110: 8284-8289

19. Yoshida S, Cui S, Ichihashi Y, Shirasu K (2016) The haustorium, a specialized invasive organ in parasitic plants. Annu Reu Plant Biol 67: 643-667

20. Conn CE, Bythell-Douglas R, Neumann D, Yoshida S, Whittington B, Westwood JH, Shirasu K, Bond CS, Dyer KA, Nelson DC (2015) PLANT EVOLUTION. Convergent evolution of strigolactone perception enabled host detection in parasitic plants. Science 349: 540-543

21. Tsuchiya Y, Yoshimura M, Sato Y, Kuwata K, Toh S, Holbrook-Smith D, Zhang H, McCourt P, Itami K, Kinoshita T et al (2015) PARASITIC PLANTS, Probing strigolactone receptors in Striga hermonthica with fluorescence. Science 349: 864-868

22. Toh S, Holbrook-Smith D, Stogios PJ, Onopriyenko O, Lumba S, Tsuchiya Y, Savchenko A, McCourt P (2015) Structure-function analysis identifies highly sensitive strigolactone receptors in Striga. Science 350: 203-207

23. Hearne SJ (2009) Control-the Striga conundrum. Pest Manag Sci 65: 603-614

24. Zwanenburg B, Mwakaboko AS, Kannan C (2016) Suicidal germination for parasitic weed control. Pest Manag Sci 72: 2016-2025
25. Jamil M, Kountche BA, Haider I, Guo X, Ntui VO, Jia KP, Ali S, Hameed US, Nakamura H, Lyu Y et al (2018) Methyl phenlactonoates are efficient strigolactone analogs with simple structure. J Exp Bot 69: $2319-2331$

26. Holbrook-Smith D, Toh S, Tsuchiya Y, McCourt P (2016) Small-molecule antagonists of germination of the parasitic plant Striga hermonthica. Nat Chem Biol 12: $724-729$

27. Xiang $H$, Yao R, Quan $T$, Wang $F$, Chen L, Du X, Zhang W, Deng H, Xie D, Luo $T$ (2017) Simple beta-lactones are potent irreversible antagonists for strigolactone receptors. Cell Res 27: 1525-1528

28. Lumba S, Holbrook-Smith D, McCourt P (2017) The perception of strigolactones in vascular plants. Nat Chem Biol 13: 599-606

29. Pouvreau JB, Gaudin Z, Auger B, Lechat MM, Gauthier M, Delavault P, Simier $P$ (2013) A high-throughput seed germination assay for root parasitic plants. Plant Methods 9: 32

30. Jeltsch A, Lanio T (2002) Site-directed mutagenesis by polymerase chain reaction. Methods Mol Biol 182: 85-94

31. Jamil M, Charnikhova T, Houshyani B, van Ast A, Bouwmeester HJ (2012) Genetic variation in strigolactone production and tillering in rice and its effect on Striga hermonthica infection. Planta 235: 473-484

32. Pai MY, Lomenick B, Hwang H, Schiestl R, McBride W, Loo JA, Huang J (2015) Drug affinity responsive target stability (DARTS) for small-molecule target identification. Methods Mol Biol 1263: 287-298

33. Franke D, Petoukhov MV, Konarev PV, Panjkovich A, Tuukkanen A, Mertens HDT, Kikhney AG, Hajizadeh NR, Franklin JM, Jeffries CM et al (2017) ATSAS 2.8: a comprehensive data analysis suite for small-angle scattering from macromolecular solutions. J Appl Crystallogr 50: $1212-1225$

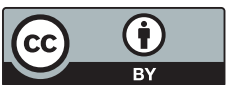

License: This is an open access article under the terms of the Creative Commons Attribution 4.0 License, which permits use, distribution and reproduction in any medium, provided the original work is properly cited. 